\title{
Minimization properties of Hill's orbits and applications to some $N$-body problems *
}

by

\author{
Gianni ARIOLI ${ }^{\mathrm{a}, 1}$, Filippo GAZZOLA ${ }^{\mathrm{a}, 2}$, Susanna TERRACINI ${ }^{\mathrm{b}, 3}$

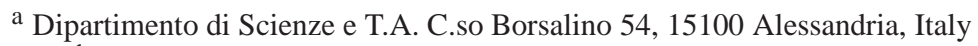 \\ b Dipartimento di Matematica, Via Bonardi 9, 20133 Milano, Italy \\ Manuscript received 11 November 1999, revised 22 February 2000
}

ABSTRACT. - We consider the periodic problem for a class of planar $\mathrm{N}$-body systems in Celestial Mechanics. Our goal is to give a variational characterization of the Hill's (retrograde) orbits as minima of the action functional under some geometrical and topological constraints. The method developed here also turns out to be useful in the study of the full problem with $N$ primaries each having at most two satellites.

(C) 2000 L'Association Publications de l'Institut Henri Poincaré. Published by Elsevier B.V. All rights reserved

Key words: $N$-body problem, Non-collision orbits

AMS classification: 70F10, 47J30

RÉSUMÉ. - On considère le problème périodique pour une certaine classe de systèmes de $N$-corps en Mécanique Céleste. Notre but est de donner une caractérisation variationnelle des orbites (rétrogrades) de Hill comme minima de la fonctionnelle d'action sous certaines contraintes géométriques et topologiques. La méthode ici développée est également

\footnotetext{
* This research was supported by MURST project "Metodi variazionali ed Equazioni Differenziali Non Lineari”.

${ }^{1}$ E-mail: gianni@mfn.unipmn.it.

${ }^{2}$ E-mail: gazzola@mfn.unipmn.it.

${ }^{3}$ E-mail: suster@mate.polimi.it.
} 
utile pour l'étude du problème complet avec $N$ corps primaires ayant chacun au plus deux satellites.

(C) 2000 L'Association Publications de l'Institut Henri Poincaré. Published by Elsevier B.V. All rights reserved

\section{INTRODUCTION}

This paper concerns the periodic problem for a class of planar $\mathrm{N}$ body systems in Celestial Mechanics. We mainly deal with a class of 3-body problems, though our method may be applied in some particular cases of more general $\mathrm{N}$-body systems. We consider the case of two major bodies and a satellite and we seek orbits when the whole system revolves with a frequence $\theta$, while the third mass rotates around one of the other bodies with a $T$-periodic motion. In the restricted case, when the motion of the satellite takes place close to one of the primaries, this problem is known as Hill's problem and a simple argument based on the inverse function theorem shows the existence of periodic orbits (in the rotating frame) for small values of the quantity $\theta T$, see [12]. Our goal is to give a variational characterization of the Hill's (retrograde) orbits as minima of the action functional under some geometrical and topological constraints. The method developed here also turns out to be useful in the study of the (full) problem with $N$ primaries each having at most two satellites.

The periodic problem for both restricted and full $N$-body systems has such a long story that it is impossible to give an extensive bibliography here; we refer the reader to the classical texts [12-14]. In the last two decades, a new method for finding periodic motions has been provided by the use of variational techniques, see, e.g., the book [3] and the references therein. The first variational characterization of the periodic solutions of the 2-body problem goes back to a paper by Gordon [11], where it is shown that the periodic orbits are local minima of the action under the topological constraint of non triviality of the rotation index. This constraint is used to overcome the lack of coercivity of the action integral in the space of periodic functions. However, from the functional point of view, the minimization problem (even in a local sense) is degenerate, that is it possesses a continuum of solutions. This is due to the fact that every solution to the associated differential equation is periodic, provided its energy is negative, and the period (and the associated action 
value) depends only on the energy. In particular all the periodic orbits, including the degenerate ellipses, where the two bodies collide, share the same variational characterization; in other words, it is impossible to distinguish them by looking at their functional levels. On the other hand, even though they can be extended as global solutions to the differential equations, the motions of collision type are periodic only in a mathematical sense.

Starting from the subsequent paper by Gordon [10], different kinds of assumptions have been considered in order to rule out the collision solutions, in the case of 2-body and $\mathrm{N}$-body problems. In the case of Keplerian interaction potentials, a fundamental remark is that the minimization problem may become non degenerate by imposing further symmetry constraints in the space of periodic functions. This fact has been first pointed out in the 2-body case in [9], and then exploited in order to obtain noncollision periodic orbits in various situations $[4,6-8,16,17]$. In the planar $N$-body problem this idea has led to associate the boundary condition $x_{i}(t+\tau)=R_{\varphi} x_{i}(t)$ with the equations system, where $x_{i}(t)$ is the position of the $i$-mass, and $R_{\varphi}$ a rotation of the plane of angle $\varphi$ and $\tau$ is the period of the mutual distances between the bodies see [4]. In this setting, it can be shown (see [5]) that the simple minimization argument in the space of symmetric functions leads to the relative equilibrium motions that are well known periodic solutions to the system [1]. In order to avoid such a triviality, Bessi and Coti Zelati [4] imposed a further topological constraint, that is one of the body couples has a non trivial rotation index in the rotating frame. However, though they ruled out the simultaneous collisions of the whole system, they were unable to avoid periodic solutions having partial collisions. The aim of this paper is to go further in the analysis in [4], and prove, by level estimates, that the minimum of the action functional under both symmetry and topological constraints is free of any collision. We first deal with the 3-body problem in the restricted and full cases: to this end, we exploit the variational structure of the problem, looking for minimizers of the action integral among the functions which satisfy the above mentioned constraints. More precisely, we require the system coordinates $X(t)=\left(x_{1}(t), x_{2}(t), x_{3}(t)\right)$ to satisfy $x_{i}(t+T)=R_{\theta T} x_{i}(t)$, where $R_{\theta T}$ is a rotation of angle $\theta T$ in the plane; in addition we require the motion of $R_{-\theta t} x_{3}(t)$ to have a negative rotation index with respect to the first body, that is, to be retrograde. Finally, we use similar arguments to study more general problems with more major bodies each one having at most two retrograde satellites. 


\section{STATEMENT OF THE RESULTS}

Throughout this paper we denote by $G$ the universal gravitation constant, by $B_{1}, \ldots, B_{n}(n=3$ or $n=4)$ the bodies of the problem we consider and by $m_{1}, \ldots, m_{n}$ their respective masses; their positions in the plane $\mathbb{R}^{2}$ are described by the $n$ functions $x_{i}=x_{i}(t)(i=1, \ldots, n)$.

DEFINITION 1. - We say that $\left(\bar{x}_{1}, \ldots, \bar{x}_{n}\right)$ is a noncollision orbit on the interval $[0, T]$ if $\bar{x}_{i}(t) \neq \bar{x}_{j}(t)$ for all $1 \leqslant i<j \leqslant n$ and all $t \in[0, T]$.

We first deal with a restricted 3-body problem $\left\{B_{1}, B_{2}, B_{3}\right\}$, which we briefly describe. Consider for a moment only the system $\left\{B_{1}, B_{2}\right\}$ : if we assume the center of mass to be fixed in the origin and we set the period to be $2 \pi / \vartheta$, then a solution of the following equations of motion

$$
-m_{i} \ddot{x}_{i}=G m_{1} m_{2} \frac{x_{i}-x_{j}}{\left|x_{i}-x_{j}\right|^{3}}, \quad x_{i}(0)=x_{i}\left(\frac{2 \pi}{\vartheta}\right), \quad i, j=1,2, i \neq j,
$$

is given by

$$
x_{1}(t)=-R_{1}(\cos \vartheta t, \sin \vartheta t), \quad x_{2}(t)=R_{2}(\cos \vartheta t, \sin \vartheta t),
$$

where

$$
\begin{aligned}
& R_{1}=m_{2} G^{1 / 3}\left(m_{1}+m_{2}\right)^{-2 / 3} \vartheta^{-2 / 3}, \\
& R_{2}=m_{1} G^{1 / 3}\left(m_{1}+m_{2}\right)^{-2 / 3} \vartheta^{-2 / 3} .
\end{aligned}
$$

The restricted problem we consider consists in assigning $x_{1}(t), x_{2}(t)$ as in (2.1), while the motion of $B_{3}$ satisfies the equation

$$
-m_{3} \ddot{x}_{3}=V^{\prime}\left(x_{3}\right)
$$

where

$$
V\left(x_{3}\right)=-\frac{G m_{1} m_{3}}{\left|x_{3}-x_{1}\right|}-\frac{G m_{2} m_{3}}{\left|x_{3}-x_{2}\right|} .
$$

Fix $T, \vartheta>0$ so that $\vartheta T<2 \pi$. Let $R_{\alpha}$ be the rotation in $\mathbb{R}^{2}$ by an angle $\alpha$; we denote by $R_{\alpha}$ the corresponding matrix as well

$$
R_{\alpha}=\left[\begin{array}{cc}
\cos \alpha & -\sin \alpha \\
\sin \alpha & \cos \alpha
\end{array}\right]
$$


Note that $x_{1}(t)=R_{\vartheta t} x_{1}(0)$ and $x_{2}(t)=R_{\vartheta t} x_{2}(0)$. We look for solutions of (2.2) satisfying $x_{3}(T)=R_{\vartheta} x_{3}(0)$; to this end we introduce the space

$$
H=\left\{x \in H^{1}\left([0, T], \mathbb{R}^{2}\right): x(T)=R_{\vartheta T} x(0)\right\} .
$$

Moreover, we require $B_{3}$ to orbit around $B_{1}$ without colliding with neither $B_{1}$ nor $B_{2}$; more precisely we consider the noncollision set

$$
\Lambda_{0}=\left\{x \in H: x(t) \neq x_{i}(t)(i=1,2) \text { for all } t \in[0, T]\right\}
$$

and

$$
\Lambda_{1}=\left\{x \in \Lambda_{0}: \operatorname{ind}\left(x-x_{1}\right) \neq 0\right\},
$$

where ind $(y)$ denotes the winding number of $y$ in the interval $[0, T]$. Consider the following Lagrangian functional

$$
L(x)=\int_{0}^{T} \frac{m_{3}}{2}|\dot{x}|^{2}-V(x),
$$

whose critical points correspond to solutions of (2.2). Then, we have

THEOREM 1.- There exists a continuum of periodic and quasiperiodic noncollision solutions of (2.2). More precisely, there exists $\bar{v} \in$ $(0,2 \pi)$ (depending only on the ratio $\left.m_{2} / m_{1}\right)$ such that if $\vartheta T \leqslant \bar{v}$ then problem (2.2) admits a solution $x_{3} \in \Lambda_{1}$; moreover, $x_{3}$ minimizes $L$ over $\Lambda_{1}$.

We prove Theorem 1 in Section 3. Of course, a major problem concerning the statement of Theorem 1 is to estimate $\bar{v}$ : in order to show that our results are not perturbative we take the masses of $B_{1}, B_{2}$ and $B_{3}$ to be respectively the masses of the Earth, the Sun and the Moon. In this case $m_{2} / m_{1} \approx(3.3) 10^{5}$ : moreover, the real (direct) motion of this 3-body system has the corresponding $\vartheta T \approx 0.46$; hence, it is of some interest to show that our method avoids collisions (for the retrograde motion) when $\bar{v}=0.46$. In fact, our next result states that even larger values are allowed:

THEOREM 2. - Assume that $m_{2} / m_{1}=(3.3) 10^{5}$; then, if $\vartheta T \leqslant 0.8$ problem (2.2) admits a solution $x_{3} \in \Lambda_{1}$; moreover, $x_{3}$ minimizes $L$ over $\Lambda_{1}$. 
This result is proved in Section 6 where we also give the pictures of some numerical experiments: such results lead us to conjecture that the upper bound for $v$ could be even larger.

Next we consider a full planar 3-body problem $\left\{B_{1}, B_{2}, B_{3}\right\}$; their motion is described by the equations

$$
-m_{i} \ddot{x}_{i}=V_{i}(x), \quad i=1,2,3,
$$

where the potential is given by

$$
V(x)=-\sum_{1 \leqslant i<j \leqslant 3} \frac{G m_{i} m_{j}}{\left|x_{j}-x_{i}\right|}
$$

and $V_{i}(x)=\partial V(x) / \partial x_{i}$. Consider the Hilbert space

$$
H=\left\{x=\left(x_{1}, x_{2}, x_{3}\right), x_{i} \in H^{1}\left([0, T], \mathbb{R}^{2}\right): x_{i}(T)=R_{\vartheta T} x_{i}(0\},\right.
$$

the noncollision set

$$
\Lambda_{0}=\left\{x \in H: x_{i}(t) \neq x_{j}(t)(1 \leqslant i<j \leqslant 3) \text { for all } t \in[0, T]\right\}
$$

and

$$
\Lambda_{1}=\left\{x \in \Lambda_{0}: \operatorname{ind}\left(x_{3}-x_{1}\right) \neq 0\right\} .
$$

By adding the three equations in (2.4) we get $\sum_{i} m_{i} \ddot{x}_{i}(t)=0$, therefore, without loss of generality we may seek solutions $x=\left(x_{1}, x_{2}, x_{3}\right) \in H$ of (2.4) which satisfy the constraint $\sum_{i} m_{i} x_{i}(t) \equiv 0$; in particular, this implies that the "interesting" degrees of freedom of the system are 4 . The Lagrangian of this 3-body problem is

$$
\Phi(x)=\int_{0}^{T} \sum_{i=1}^{3} \frac{m_{i}}{2}\left|\dot{x}_{i}\right|^{2}-V(x) .
$$

Also in this case we obtain infinitely many noncollision periodic or quasi-periodic solutions of (2.4): in Section 4 we prove

THEOREM 3. - If $m_{3}$ is sufficiently small, then there exists a continuum of periodic and quasi-periodic noncollision solutions of (2.4). More precisely, there exist two constants $\bar{v} \in(0,2 \pi)$ and $M>0$ depending only on $m_{1}, m_{2}$ such that if $\vartheta T \leqslant \bar{v}$ and $m_{3} \leqslant M$, then problem (2.4) admits a solution $x \in \Lambda_{1}$; moreover, $x$ minimizes $\Phi$ over $\Lambda_{1}$. 
This result enables us to study the planar 4-body problem $\left\{B_{1}, B_{2}, B_{3}\right.$, $\left.B_{4}\right\}$ where $B_{3}$ and $B_{4}$ are two satellites of $B_{1}$. Their motion is described by the equations

$$
-m_{i} \ddot{x}_{i}=V_{i}(x), \quad i=1,2,3,4,
$$

where the potential is given by

$$
V(x)=-\sum_{1 \leqslant i<j \leqslant 4} \frac{G m_{i} m_{j}}{\left|x_{j}-x_{i}\right|} .
$$

Define the Hilbert space

$$
H=\left\{x=\left(x_{1}, x_{2}, x_{3}, x_{4}\right), x_{i} \in H^{1}\left([0, T], \mathbb{R}^{2}\right): x_{i}(T)=R_{\vartheta T} x_{i}(0)\right\},
$$

the noncollision set

$$
\Lambda_{0}=\left\{x \in H: x_{i}(t) \neq x_{j}(t)(1 \leqslant i<j \leqslant 4) \text { for all } t \in[0, T]\right\},
$$

and

$$
\Lambda_{1}=\left\{x \in \Lambda_{0}: \operatorname{ind}\left(x_{i}-x_{1}\right) \neq 0, i=3,4\right\} .
$$

The corresponding Lagrangian is

$$
\Phi(x)=\int_{0}^{T} \sum_{i=1}^{4} \frac{m_{i}}{2}\left|\dot{x}_{i}\right|^{2}-V(x) .
$$

Then, in Section 5 we will prove

THEOREM 4. - If $m_{3}$ and $m_{4}$ are sufficiently small, then there exists a continuum of periodic and quasi-periodic noncollision solutions of (2.6). More precisely, there exist two constants $\bar{v} \in(0,2 \pi)$ and $M>0$ depending only on $m_{1}, m_{2}$ such that if $\vartheta T \leqslant \bar{v}$ and $m_{3}, m_{4} \leqslant M$, then problem (2.6) admits a solution $x \in \Lambda_{1}$; moreover, $x$ minimizes $\Phi$ over $\Lambda_{1}$.

Remark 1. - The proofs of our results may be naturally modified in order to obtain similar statements for $N$-body problems $\left\{B_{1}, \ldots, B_{N}\right\}$ with $k$ major bodies $(k<N)$, each one having at most two satellites.

Remark 2. - Although we seek solutions in $\Lambda_{1}$ (a set having nontrivial index for some couples of bodies), in fact by construction all the solutions 
we find have the index equal to -1 , therefore they represent a clockwise motion, while the two major bodies are assumed to rotate anticlockwise.

\section{THE RESTRICTED PROBLEM}

In this section we prove Theorem 1.

First assume that $\vartheta T<2 \pi$. We switch to a rotating coordinate frame $\left(0, e_{1}, e_{2}\right)$ so that the bodies $B_{1}$ and $B_{2}$, whose motions in the original coordinate frame are described by (2.1), are at rest. More precisely, the position of $B_{1}$ is $-R_{1} e_{1}$ and the position of $B_{2}$ is $R_{2} e_{1}$. If we set $q(t)=R_{-\vartheta t} x_{3}(t)$ and if

$$
J=\left(\begin{array}{cc}
0 & -1 \\
1 & 0
\end{array}\right)
$$

denotes the standard symplectic matrix, then we get $\dot{x}_{3}(t)=\dot{R}_{\vartheta t} q(t)+$ $R_{\vartheta t} \dot{q}(t)$ and $\left|\dot{x}_{3}(t)\right|^{2}=|\dot{q}(t)+\vartheta J q(t)|^{2}$; the condition $x_{3}(T)=R_{\vartheta} x_{3}(0)$ becomes $q(T)=q(0)$. Next we rescale the period $T$ and we translate the system in order to have $B_{1}$ at the origin by setting $y(t / T)=q(t)+R_{1} e_{1}$, so that we get the standard Lagrangian functional of the restricted 3-body problem:

$$
I(y)=\frac{1}{T} \int_{0}^{1} \frac{m_{3}}{2}\left|\dot{y}+T \vartheta J\left(y-R_{1} e_{1}\right)\right|^{2}-T^{2} V(y),
$$

where

$$
V(y)=-\frac{G m_{1} m_{3}}{|y|}-\frac{G m_{2} m_{3}}{\left|y-\left(R_{1}+R_{2}\right) e_{1}\right|} .
$$

Up to the addition of a constant, we may redefine the Lagrangian $I$ as follows:

$$
I(y)=\frac{1}{T} \int_{0}^{1} \frac{m_{3}}{2}|\dot{y}+T \vartheta J y|^{2}-m_{3} \vartheta^{2} T^{2} R_{1}\left(y, e_{1}\right)-T^{2} V(y)
$$

we remark that the corresponding Euler-Lagrange equations are unchanged. We set $x(t)=\left(T^{2} G m_{1}\right)^{-1 / 3} y(t)$, we introduce the adimen- 
sional parameters $\rho=m_{2} / m_{1}$ and $\nu=\vartheta T$ and we define

$$
\Phi(x)=m_{3}^{-1} T^{-1 / 3}\left(G m_{1}\right)^{-2 / 3} I(x)-\frac{\rho v^{2 / 3}}{(\rho+1)^{1 / 3}},
$$

so that we get

$$
\begin{aligned}
\Phi(x)= & \int_{0}^{1} \frac{1}{2}|\dot{x}+T \vartheta J x|^{2}+\frac{1}{|x|} \\
& +\frac{T^{2 / 3} G^{1 / 3} m_{2} m_{1}^{-2 / 3}}{\left|\left(T^{2} G m_{1}\right)^{1 / 3} x-\left(m_{1}+m_{2}\right)^{1 / 3} G^{1 / 3} \vartheta^{-2 / 3} e_{1}\right|} \\
& -\frac{\vartheta^{2} T^{4 / 3}}{\left(G m_{1}\right)^{1 / 3}} \frac{m_{2} G^{1 / 3}}{\left(m_{1}+m_{2}\right)^{2 / 3} \vartheta^{2 / 3}}\left(x, e_{1}\right)-\frac{\rho v^{2 / 3}}{(\rho+1)^{1 / 3}} \\
= & \int_{0}^{1} \frac{1}{2}|\dot{x}+T \vartheta J x|^{2}+\frac{1}{|x|}+\frac{m_{2} / m_{1}}{\left|x-\left(m_{2} / m_{1}+1\right)^{1 / 3}(\vartheta T)^{-2 / 3} e_{1}\right|} \\
& -\frac{\vartheta^{4 / 3} T^{4 / 3} m_{2}}{m_{1}^{1 / 3}\left(m_{1}+m_{2}\right)^{2 / 3}}\left(x, e_{1}\right)-\frac{\rho v^{2 / 3}}{(\rho+1)^{1 / 3}} \\
= & \int_{0}^{1} \frac{1}{2}|\dot{x}+v J x|^{2}+\frac{1}{|x|}+\frac{\rho}{\left|x-(\rho+1)^{1 / 3} v^{-2 / 3} e_{1}\right|} \\
& -\frac{\rho v^{4 / 3}}{(\rho+1)^{2 / 3}}\left(x, e_{1}\right)-\frac{\rho v^{2 / 3}}{(\rho+1)^{1 / 3}} .
\end{aligned}
$$

For all $\xi \in \mathbb{R}^{2}$ define the functions

$$
g(\xi):=\frac{\rho}{\left|\xi-(\rho+1)^{1 / 3} v^{-2 / 3} e_{1}\right|}
$$

and

$$
f(\xi):=g(\xi)-\frac{\rho v^{2 / 3}}{(\rho+1)^{1 / 3}}-\frac{\rho v^{4 / 3}}{(\rho+1)^{2 / 3}}\left(\xi, e_{1}\right)
$$

so that

$$
\Phi(x)=\int_{0}^{1} \frac{1}{2}|\dot{x}+v J x|^{2}+\frac{1}{|x|}+f(x) .
$$

Note that 


$$
\begin{gathered}
\nabla g(\xi)=-\rho \frac{\xi-(\rho+1)^{1 / 3} v^{-2 / 3} e_{1}}{\left|\xi-(\rho+1)^{1 / 3} v^{-2 / 3} e_{1}\right|^{3}}, \\
\nabla^{2} g(\xi)[\zeta, \zeta]=- \\
+\frac{\rho|\zeta|^{2}}{\left|\xi-(\rho+1)^{1 / 3} v^{-2 / 3} e_{1}\right|^{3}} \\
+\frac{3 \rho\left(\xi-(\rho+1)^{1 / 3} v^{-2 / 3} e_{1}, \zeta\right)^{2}}{\left|\xi-(\rho+1)^{1 / 3} v^{-2 / 3} e_{1}\right|^{5}}
\end{gathered}
$$

and, by expanding in McLaurin polynomial, there exists $s \in(0,1)(s=$ $s(\xi))$ such that

$$
\begin{aligned}
g(\xi)= & \frac{\rho v^{2 / 3}}{(\rho+1)^{1 / 3}}+\frac{\rho v^{4 / 3}}{(\rho+1)^{2 / 3}}\left(\xi, e_{1}\right)-\frac{\rho|\xi|^{2}}{2\left|s \xi-(\rho+1)^{1 / 3} v^{-2 / 3} e_{1}\right|^{3}} \\
& +\frac{3 \rho\left(s \xi-(\rho+1)^{1 / 3} v^{-2 / 3} e_{1}, \xi\right)^{2}}{2\left|s \xi-(\rho+1)^{1 / 3} v^{-2 / 3} e_{1}\right|^{5}}
\end{aligned}
$$

therefore, we also have for some $s=s(\xi) \in(0,1)$

$$
\begin{aligned}
f(\xi)= & -\frac{\rho|\xi|^{2}}{2\left|s \xi-(\rho+1)^{1 / 3} v^{-2 / 3} e_{1}\right|^{3}} \\
& +\frac{3 \rho\left(s \xi-(\rho+1)^{1 / 3} v^{-2 / 3} e_{1}, \xi\right)^{2}}{2\left|s \xi-(\rho+1)^{1 / 3} v^{-2 / 3} e_{1}\right|^{5}} .
\end{aligned}
$$

The Lagrangian functional $L$ defined in (2.3) is therefore transformed into $\Phi$ while the original space $H$ is transformed in the Hilbert space $H_{1}^{1}$ of 1-periodic functions in $H^{1}$. Consider the noncollision open subset

$$
\Lambda_{0}=\left\{x \in H_{1}^{1}: x(t) \neq 0 \text { and } x(t) \neq(\rho+1)^{1 / 3} v^{-2 / 3} e_{1} \text { for all } t \in[0,1]\right\}
$$

and

$$
\Lambda_{1}=\left\{x \in \Lambda_{0}: \operatorname{ind}(x)=-1\right\} .
$$

Theorem 1 is proved if we show that the problem

$$
\inf _{x \in \Lambda_{1}} \Phi(x)
$$

admits a solution $x \in \Lambda_{1}$ and no solutions in $\partial \Lambda_{1}$.

LEMMA 1. - Let $\left\{x^{n}\right\} \subset \Lambda_{1}$ be a minimizing sequence for $\Phi$; then there exists $\underline{x} \in \bar{\Lambda}_{1}$ such that $x^{n} \rightarrow \underline{x}$, up to a subsequence, and $\Phi(\underline{x})=$ $\inf _{x \in \Lambda_{1}} \Phi(x)$.

Proof. - In the following $c$ represents a generic positive constant which may vary within the same formula. Let $x_{0}=R(\sin 2 \pi t, \cos 2 \pi t)$ with 
$R=(2 \pi)^{-2 / 3}$; then $x_{0}$ achieves the minimum of the functional

$$
\Psi(x)=\int_{0}^{1} \frac{|\dot{x}|^{2}}{2}+\frac{1}{|x|}
$$

under the constraint ind $(x)=-1 ; x_{0}$ is not a minimum of $\Phi$, therefore for large values of $n$ we have $\Phi\left(x^{n}\right) \leqslant \Phi\left(x_{0}\right)$ and

$$
\int_{0}^{1} \frac{1}{2}\left|\dot{x}^{n}+v J x^{n}\right|^{2}-c\left(x^{n}, e_{1}\right) \leqslant \Phi\left(x_{0}\right)+\frac{\rho v^{2 / 3}}{(\rho+1)^{1 / 3}}=c .
$$

Note that if $v \leqslant \bar{v}$ then

$$
\inf _{\|\dot{x}\|_{2}=1} \int_{0}^{1}|\dot{x}+v J x|^{2}=\left(1-\frac{v}{2 \pi}\right)^{2} \geqslant\left(1-\frac{\bar{v}}{2 \pi}\right)^{2}
$$

so the last inequality yields

$$
c\left\|\dot{x}^{n}\right\|_{2}^{2}-c\left\|\dot{x}^{n}\right\|_{2} \leqslant c
$$

and finally $\left\|\dot{x}^{n}\right\|_{2} \leqslant c$. By the topological requirements and Poincaré inequality we infer $\left\|x^{n}\right\|_{\infty} \leqslant c\left\|\dot{x}^{n}\right\|_{2} \leqslant c$, therefore $\left\{x^{n}\right\}$ is bounded in $H$ and, up to a subsequence, it admits a weak limit $\underline{x} \in H$, which is a minimum of $\Phi$ by the weak lower semicontinuity of the functional $\Phi$. Finally, $\underline{x} \in \bar{\Lambda}_{1}$ by uniform convergence of $\left\{x^{n}\right\}$.

To complete the proof of Theorem 1 we exclude the case $\underline{x} \in \partial \Lambda_{1}$ in the statement of the previous lemma; first of all it is possible to obtain a contradiction to a collision between $B_{3}$ and $B_{2}$, because they are not linked by a topological constraint. Indeed, if there exists $\tau \in[0,1]$ such that $\underline{x}(\tau)=(\rho+1)^{1 / 3} v^{-2 / 3} e_{1}$, we can modify the trajectory of $\underline{x}=\underline{x}(t)$ in a neighborhood of $\tau$ in order to lower both the kinetic part and the potential part of $\Phi$ and to avoid a collision. Since the proof is standard, we omit it.

LEMMA 2. - Let $\underline{x}$ be as in Lemma 1 ; then $\underline{x}(t) \neq(\rho+1)^{1 / 3} v^{-2 / 3} e_{1}$ for all $t \in[0,1]$.

The proof that $B_{3}$ does not collide with $B_{1}$ is more subtle and requires some estimates; we exclude collisions for sufficiently small $v$ : 
LEMma 3. - Let $\underline{x}$ denote an orbit obtained in Lemma 1. There exists $\bar{v} \in(0,2 \pi)$ such that if $v \leqslant \bar{v}$ then $\underline{x}(t) \neq 0$ for all $t \in[0,1]$ and

$$
\inf _{\Lambda_{1}} \Phi<\inf _{\partial \Lambda_{1}} \Phi
$$

in particular, $\underline{x} \in \Lambda_{1}$.

Proof. - In this proof we denote by $K_{i}(i=1, \ldots, 4)$ positive constants depending (eventually) on $\rho$. By contradiction, assume that $\underline{x} \in \partial \Lambda_{1}$, where $\underline{x}$ is determined by the statement of Lemma 1; without loss of generality, we may assume that $\underline{x}(0)=0$ and hence

$$
\|\underline{x}\|_{2} \leqslant \frac{1}{\pi}\|\underline{\dot{x}}\|_{2}, \quad\|\underline{x}\|_{\infty} \leqslant \frac{1}{2}\|\underline{\dot{x}}\|_{2} .
$$

Consider again the function $x_{0}=R(\sin 2 \pi t, \cos 2 \pi t)$ with $R=(2 \pi)^{-2 / 3}$; if $v$ is sufficiently small, then we have $\left|s x_{0}(t)-(\rho+1)^{1 / 3} v^{-2 / 3} e_{1}\right| \geqslant$ $\frac{1}{2}(\rho+1)^{1 / 3} v^{-2 / 3}$ for all $t \in[0,1]$ and $s \in(0,1)$, hence, (3.1) yields

$$
f\left(x_{0}\right) \leqslant \frac{\rho R^{2}}{2\left|s x_{0}-(\rho+1)^{1 / 3} v^{-2 / 3} e_{1}\right|^{3}} \leqslant K_{1} v^{2}, \quad \forall t \in[0,1],
$$

and

$$
\Phi\left(x_{0}\right) \leqslant \frac{(2 \pi-v)^{2}}{2(2 \pi)^{4 / 3}}+(2 \pi)^{2 / 3}+K_{1} v^{2}<\frac{3}{2}(2 \pi)^{2 / 3}-K_{2} v+K_{3} v^{2} .
$$

Since $\underline{x}$ minimizes $\Phi$, by (3.3) (3.4) and (3.5) we get

$$
\begin{aligned}
& \frac{1}{2}\left(1-\frac{v}{2 \pi}\right)^{2}\|\underline{\dot{x}}\|_{2}^{2}-\frac{\rho v^{4 / 3}}{\pi(\rho+1)^{2 / 3}}\|\underline{\dot{x}}\|_{2}-\frac{\rho v^{2 / 3}}{(\rho+1)^{1 / 3}} \\
& \quad \leqslant \Phi(\underline{x}) \leqslant \Phi\left(x_{0}\right)<\frac{3}{2}(2 \pi)^{2 / 3}-K_{2} v+K_{3} v^{2} ;
\end{aligned}
$$

therefore, we obtain (independently of $v \leqslant \bar{v} \leqslant \pi$ ) $\|\underline{\dot{x}}\|_{2} \leqslant K_{4}$ : in turn, if $v$ is sufficiently small, by (3.4) this yields

$$
\|\underline{x}\|_{\infty} \leqslant \frac{1}{2} \frac{(\rho+1)^{1 / 3}}{v^{2 / 3}} .
$$


On the other hand, by (3.1)

$$
\Phi(\underline{x}) \geqslant \int_{0}^{1} \frac{1}{2}|\underline{\dot{x}}+v J \underline{x}|^{2}+\frac{1}{|\underline{x}|}-\frac{\rho|\underline{x}|^{2}}{2\left|s \underline{x}-(\rho+1)^{1 / 3} v^{-2 / 3} e_{1}\right|^{3}}
$$

which, together with (3.6), gives

$$
\Phi(\underline{x}) \geqslant \int_{0}^{1} \frac{1}{2}|\underline{\dot{x}}+v J \underline{x}|^{2}+\frac{1}{|\underline{x}|}-4 v^{2}|\underline{x}|^{2}
$$

finally, note that

$$
\begin{gathered}
\inf _{x \in H_{0}^{1}} \int_{0}^{1} \frac{1}{2}|\dot{x}+v J x|^{2}+\frac{1}{|x|}-4 v^{2}|x|^{2} \\
=\inf _{x \in H_{0}^{1}} \int_{0}^{1} \frac{1}{2}|\dot{x}|^{2}+\frac{1}{|x|}-4 v^{2}|x|^{2} .
\end{gathered}
$$

Therefore, since we assume that $\underline{x} \in H_{0}^{1}$ minimizes $\Phi$, by (3.4), (3.5), (3.7) and (3.8)

$$
\begin{aligned}
& \frac{3}{2}(2 \pi)^{2 / 3}-K_{2} v+K_{3} v^{2} \\
& >\Phi\left(x_{0}\right) \geqslant \Phi(\underline{x}) \geqslant \inf _{x \in H_{0}^{1}} \int_{0}^{1} \frac{1}{2}|\dot{x}|^{2}+\frac{1}{|x|}-4 v^{2}|x|^{2} \\
& \geqslant \inf _{y \in H_{0}^{1}} \int_{0}^{1} \frac{1}{2}\left(1-\frac{8 v^{2}}{\pi^{2}}\right)|\dot{y}|^{2}+\frac{1}{|y|} \\
& \quad=\left(1-\frac{8 v^{2}}{\pi^{2}}\right)^{1 / 3} \inf _{z \in H_{0}^{1}} \int_{0}^{1} \frac{1}{2}|\dot{z}|^{2}+\frac{1}{|z|}=\left(1-\frac{8 v^{2}}{\pi^{2}}\right)^{1 / 3} \frac{3}{2}(2 \pi)^{2 / 3}
\end{aligned}
$$

which is impossible if $v>0$ is small enough: hence, for such $v$, we get a contradiction and the lemma is proved.

Theorem 1 is proved: if $\theta T \leqslant \bar{v}$ we obtain a solution of (2.2); such solution is periodic if $\theta T / \pi \in \mathbb{Q}$, while it is quasi-periodic if $\theta T / \pi \in$ $\mathbb{R} \backslash \mathbb{Q}$. 


\section{THE FULL 3-BODY PROBLEM}

In this section we prove Theorem 3.

To this end, we show that the Lagrangian functional $\Phi$ defined in (2.5) admits a global minimum in the (open) set

$$
\Lambda_{1}=\left\{x \in \Lambda_{0}: \operatorname{ind}\left(x_{3}-x_{1}\right)=-1\right\},
$$

where $\Lambda_{0}$ is the noncollision open set

$$
\Lambda_{0}=\left\{x \in H: x_{i}(t) \neq x_{j}(t) \text { for all } t \in[0, T] \text { and } i \neq j\right\} .
$$

We first prove

LEMMA 4. - Let $\left\{x^{n}\right\} \subset \Lambda_{1}$ be a minimizing sequence for $\Phi$; then $\left\{x^{n}\right\}$ is bounded in $H$.

Proof. - Since $\Phi(x) \geqslant \sum_{i} \int_{0}^{T} \frac{m_{i}}{2}\left|\dot{x}_{i}\right|^{2}$ for all $x \in \Lambda_{0}$, then $\left\|\dot{x}^{n}\right\|_{2}$ is bounded. Since $x_{i}(T)=R_{\vartheta T} x_{i}(0)$, then $\left|x_{i}(t+T)-x_{i}(t)\right|^{2}=\mid\left(R_{\vartheta T}-\right.$ $\left.R_{0}\right)\left.x_{i}(t)\right|^{2}=c\left|x_{i}(t)\right|^{2}$. But $x_{i}(t+T)-x_{i}(t)=\int_{t}^{t+T} \dot{x}_{i}(s) \mathrm{d} s$, hence $\left|x_{i}(t)\right|^{2} \leqslant c^{-1}\left\|\dot{x}_{i}^{n}\right\|_{1}^{2} \leqslant T c^{-1}\left\|\dot{x}_{i}^{n}\right\|_{2}^{2}$ for all $t$, so $\left\|x^{n}\right\|_{\infty}$ is bounded and finally $\left\|x^{n}\right\|$ is bounded.

We use again a rotating coordinate system by setting $Q_{i}(t)=R_{-\vartheta t} x_{i}(t)$ for all $x=\left(x_{1}, x_{2}, x_{3}\right) \in H$ so that $\dot{x}_{i}(t)=\dot{R}_{\vartheta t} Q_{i}(t)+R_{\vartheta t} \dot{Q}_{i}(t)$ and $\left|\dot{x}_{i}(t)\right|^{2}=\left|\dot{Q}_{i}(t)+\vartheta J Q_{i}(t)\right|^{2}$. Next we rescale the period by setting $y(t / T)=Q(t)$. Up to a multiplication by $T$, the Lagrangian becomes

$$
I(y)=\int_{0}^{1} \sum_{i=1}^{3} \frac{m_{i}}{2}\left|\dot{y}_{i}+T \vartheta J y_{i}\right|^{2}-T^{2} V(y)
$$

clearly, the constraint $\sum_{i} m_{i} x_{i}(t) \equiv 0$ transforms into $\sum_{i} m_{i} y_{i}(t) \equiv 0$. We introduce a new Hilbert space of periodic functions (which we still denote by $H$ ), defined by

$$
H=\left\{y=\left(y_{1}, y_{2}, y_{3}\right) \in H^{1}\left([0,1], \mathbb{R}^{6}\right): y(1)=y(0), \sum_{i=1}^{3} m_{i} y_{i}(t) \equiv 0\right\},
$$

the corresponding noncollision open set

$$
\Lambda_{0}=\left\{y \in H: y_{i}(t) \neq y_{j}(t) \text { for all } t \in[0,1] \text { and } i \neq j\right\}
$$


and its subset

$$
\Lambda_{1}=\left\{y \in \Lambda_{0}: \operatorname{ind}\left(y_{3}-y_{1}\right)=-1\right\}
$$

Therefore, the original problem of minimizing $\Phi$ is reduced to the following minimization problem:

$$
\inf _{y \in \Lambda_{1}} I(y)
$$

We prove that the infimum in (4.1) is achieved:

LEMMA 5. - There exists $\underline{y} \in \bar{\Lambda}_{1}$ such that $I(\underline{y})=\inf _{y \in \Lambda_{1}} I(y)$.

Proof. - Let $\left\{y^{n}\right\} \subset \Lambda_{1}$ be a minimizing sequence for $I$; then, the corresponding sequence $\left\{x^{n}\right\}$ defined by setting $x_{i}^{n}(t)=R_{\vartheta t} y_{i}^{n}(t / T)$ is minimizing for $\Phi$ : by Lemma 4, it is bounded and so is $\left\{y^{n}\right\}$. Therefore, up to a subsequence, $\left\{y^{n}\right\}$ admits a weak limit $y \in H$, which is a minimum of $I$ by the lower semicontinuity of the functional $I$; finally, $\underline{y} \in \bar{\Lambda}_{1}$ by uniform convergence of the sequence $\left\{y^{n}\right\}$.

As in Section 3, one can easily exclude collisions between $B_{2}$ and $B_{3}$ : we have to exclude the other possible collisions.

Let $I_{0}$ be the Lagrangian $I$ corresponding to $m_{3}=0$ : as $I_{0}$ does not depend on the third component $y_{3}$ of $y$, we may identify it with its restriction to the subspace $H_{0}$ corresponding to $y_{3}=0$, namely

$$
H_{0}=\left\{y=\left(y_{1}, y_{2}\right) \in H^{1}\left([0,1], \mathbb{R}^{4}\right): y(1)=y(0), \sum_{i=1}^{2} m_{i} y_{i}(t) \equiv 0\right\}
$$

and we study the minimization (2-body) problem

$$
\inf _{x \in \Omega_{0}} I_{0}(x)
$$

where $\Omega_{0}$ is the corresponding noncollision open set. Consider the function $f: \mathbb{R}^{4} \rightarrow \mathbb{R}$ defined by

$$
f\left(\xi_{1}, \xi_{2}\right)=\sum_{i=1}^{2} \frac{m_{i}}{2} \vartheta^{2}\left|\xi_{i}\right|^{2}+\frac{m_{1} m_{2}}{\left|\xi_{1}-\xi_{2}\right|} \quad\left(\xi_{i} \in \mathbb{R}^{2}\right)
$$

one can easily check that $f$ has a unique strict global minimum, up to a $S O$ (2)-symmetry: more precisely, there exists $\Sigma \subset \mathbb{R}^{4}$ (which is a $S O$ (2) 
orbit), such that $f$ attains its global minima on $\Sigma$. One such minimum is

$$
\vartheta^{-2 / 3}\left(-\frac{m_{2}}{\left(m_{1}+m_{2}\right)^{2 / 3}}, 0, \frac{m_{1}}{\left(m_{1}+m_{2}\right)^{2 / 3}}, 0\right)
$$

and the corresponding Hessian matrix of $f$ has rank 3 with 3 strictly positive eigenvalues (we denote by $C>0$ the smallest of these eigenvalues); the 0 eigenvalue corresponds to the direction tangent to $\Sigma$. Therefore, if we denote by $\xi^{\Sigma}=\left(\xi_{1}^{\Sigma}, \xi_{2}^{\Sigma}\right)$ the projection on $\Sigma$ of any $\xi=\left(\xi_{1}, \xi_{2}\right) \in \mathbb{R}^{4}$ sufficiently close to $\Sigma$ we have

$$
\begin{aligned}
& \sum_{i=1}^{2} \frac{m_{i}}{2} \vartheta^{2}\left|\xi_{i}\right|^{2}+\frac{m_{1} m_{2}}{\left|\xi_{1}-\xi_{2}\right|} \\
& \quad \geqslant \sum_{i=1}^{2} \frac{m_{i}}{2} \vartheta^{2}\left|\xi_{i}^{\Sigma}\right|^{2}+\frac{m_{1} m_{2}}{\left|\xi_{1}^{\Sigma}-\xi_{2}^{\Sigma}\right|}+\frac{C}{2} \sum_{i=1}^{2}\left|\xi_{i}-\xi_{i}^{\Sigma}\right|^{2}
\end{aligned}
$$

in particular, any (stationary) point in $\Sigma$ is also a minimum for the functional $I_{0}$.

Fix $\vartheta>0$ : we prove that if $m_{3}$ and $T$ are small enough and $y=$ $\left(y_{1}, y_{2}, y_{3}\right)$ is the minimum obtained in Lemma 5 , then $\left(y_{1}, y_{2}\right)$ is close to $\Sigma$ in the $H$ norm topology. In particular, this shows that $B_{1}$ and $B_{2}$ do not collide.

LEMMA 6. - There exist two constants $\bar{T}, M>0$ depending only on $\theta, m_{1}, m_{2}$ and a constant $c>0$ such that for all $T \leqslant \bar{T}$, all $m_{3} \leqslant M$ and all $y=\left(y_{1}, y_{2}, y_{3}\right) \in H$ achieving the minimum in (4.1) (as given by Lemma 5) we have

$$
\sum_{i=1}^{2}\left(\left\|\dot{y}_{i}\right\|_{2}^{2}+T^{2}\left\|y_{i}-y_{i}^{\Sigma}\right\|_{2}^{2}\right) \leqslant c T^{4 / 3} m_{3},
$$

where $y_{i}^{\Sigma}=y_{i}^{\Sigma}(t)$ is the (pointwise) projection of $y_{i}(t)$ onto $\Sigma$.

Proof. - Take any $\left(y_{E}, y_{S}\right) \in \Sigma$ and consider the function $Y \in H$ defined by

$$
\left\{\begin{array}{l}
Y_{1}(t) \equiv y_{E} \\
Y_{2}(t) \equiv y_{S} \\
Y_{3}(t)=y_{E}+T^{2 / 3}(\sin 2 \pi t, \cos 2 \pi t)
\end{array}\right.
$$

so that

$$
V_{23}\left(y_{E}, Y_{3}\right)=-\frac{m_{2} m_{3}}{T^{2 / 3}}
$$


and $\dot{Y}_{3}(t)=2 \pi T^{2 / 3}(\cos 2 \pi t,-\sin 2 \pi t)$. Then the kinetic part of $Y_{3}$ is given by

$$
\begin{aligned}
\left|\dot{Y}_{3}+T \vartheta J Y_{3}\right|^{2}= & T^{4 / 3} \mid 2 \pi(\cos 2 \pi t,-\sin 2 \pi t) \\
& +\left.T^{1 / 3} \vartheta J\left(y_{E}+T^{2 / 3}(\sin 2 \pi t, \cos 2 \pi t)\right)\right|^{2} .
\end{aligned}
$$

If $T$ is small enough, then $Y \in \Lambda_{1}$, and by taking into account that $\inf _{\Lambda_{1}} I \leqslant I(Y)$ we obtain

$$
\inf _{\Lambda_{1}} I_{0}(y) \leqslant \inf _{\Lambda_{1}} I \leqslant \inf _{\Lambda_{1}} I_{0}+c_{1} T^{4 / 3} m_{3}+c_{2} T^{2} m_{3} .
$$

Let $\left\{m_{3}^{n}\right\}$ be a vanishing sequence, let $I_{n}$ be the functional corresponding to $m_{3}^{n}$ and let $y^{n}=y^{n}\left(m_{3}^{n}, T\right) \in \bar{\Lambda}_{1}^{n}$ be the minimum of $I_{n}$ obtained in Lemma 5. If $y_{i}^{n}(i=1,2,3)$ denote the components of $y^{n}$, then by (4.4) we infer that $\left(y_{1}^{n}, y_{2}^{n}\right)$ is a minimizing sequence for $I_{0}$, hence it converges weakly in $H$ and uniformly to some $\left(y_{E}, y_{S}\right) \in \Sigma$, up to a subsequence. In particular, this proves that for any given $\varepsilon>0$ there exists $m_{3}^{\varepsilon}>0$ such that if $m_{3} \leqslant m_{3}^{\varepsilon}$, then

$$
\left|y_{1}(t)-y_{E}\right| \leqslant \varepsilon \quad \text { and } \quad\left|y_{2}(t)-y_{S}\right| \leqslant \varepsilon \quad \text { for all } t \in[0,1] \text {. }
$$

For all $y \in H^{1}\left(S^{1}, \mathbb{R}^{2}\right)$ satisfying $\int_{0}^{1} y=0$ we have $\|y\|_{\infty} \leqslant\|\dot{y}\|_{1} \leqslant$ $\gamma\|\dot{y}\|_{2}$ (the first is Wirtinger inequality and the second is Hölder's). Then

$$
\left|\int_{0}^{1}(J \dot{y}, y)\right|=\left|\int_{0}^{1}\left(J \dot{y}, y-\int_{0}^{1} y\right)\right| \leqslant\|\dot{y}\|_{2}^{2} \text {. }
$$

Now let $\bar{y}=\left(y_{E}, y_{S}\right)$, fix $y \in H^{1}\left(S^{1}, \mathbb{R}^{4}\right)$ and let $\tilde{y}=y-\bar{y}$; since $\int_{0}^{1}(J \dot{y}, \bar{y})=0$, then

$\int_{0}^{1}\left|\dot{y}_{i}+T \vartheta J y_{i}\right|^{2}=\int_{0}^{1}\left(\left|\dot{y}_{i}\right|^{2}+T^{2} \vartheta^{2}\left|\bar{y}_{i}+\tilde{y}_{i}\right|^{2}-2 T \vartheta\left(J \dot{y}_{i}, \tilde{y}_{i}\right)\right), \quad i=1,2$.

Recall that $f(\bar{y})=f\left(y^{\Sigma}(t)\right)$ for all $t \in[0,1]$, using (4.3), (4.5) and (4.6) we get:

$$
I_{0}(y)-I_{0}(\bar{y})=\int_{0}^{1} \sum_{i=1}^{2} \frac{m_{i}}{2}\left(\left|\dot{y}_{i}\right|^{2}+T^{2} \vartheta^{2}\left|y_{i}\right|^{2}-2 T \vartheta\left(J \dot{y}_{i}, \tilde{y}_{i}\right)\right)
$$




$$
\begin{aligned}
& +\frac{T^{2} m_{1} m_{2}}{\left|y_{1}-y_{2}\right|}-\int_{0}^{1} \sum_{i=1}^{2} \frac{m_{i}}{2} T^{2} \vartheta^{2}\left|y_{i}^{\Sigma}\right|^{2}+\frac{T^{2} m_{1} m_{2}}{\left|y_{1}^{\Sigma}-y_{2}^{\Sigma}\right|} \\
\geqslant & \int_{0}^{1} \sum_{i=1}^{2} \frac{m_{i}}{2}\left(\left|\dot{y}_{i}\right|^{2}-2 T \vartheta\left(J \dot{y}_{i}, \tilde{y}_{i}\right)\right)+\frac{C}{2} T^{2}\left|y_{i}-y_{i}^{\Sigma}\right|^{2} \\
\geqslant & \sum_{i=1}^{2} \frac{m_{i}}{4}\left\|\dot{y}_{i}\right\|_{2}^{2}+\frac{C}{2} T^{2}\left\|y_{i}-y_{i}^{\Sigma}\right\|_{2}^{2} ;
\end{aligned}
$$

the last inequality follows by Wirtinger inequality and (4.6) since, for $T$ small enough, we have

$$
\int_{0}^{1} \sum_{i=1}^{2} m_{i} T \vartheta\left(\dot{y}_{i}, J \tilde{y}_{i}\right) \leqslant \sum_{i=1}^{2} \frac{m_{i}}{4}\left\|\dot{y}_{i}\right\|_{2}^{2}
$$

Finally, from (4.4) we get $\inf _{\Lambda_{1}} I-\inf _{\Lambda_{1}} I_{0} \leqslant c_{1} T^{4 / 3} m_{3}+c_{2} T^{2} m_{3}$ and therefore

$$
\sum_{i=1}^{2} \frac{m_{i}}{4}\left\|\dot{y}_{i}\right\|_{2}^{2}+\frac{C}{2} T^{2}\left\|y_{i}-y_{i}^{\Sigma}\right\|_{2}^{2} \leqslant c_{1} T^{4 / 3} m_{3}+c_{2} T^{2} m_{3},
$$

which proves the estimate.

Finally, to prove that the minimum obtained in Lemma 5 is a noncollision minimum, we show that $B_{1}$ and $B_{3}$ do not collide:

LEMMA 7. - If $T$ and $m_{3}$ are sufficiently small, then

$$
\inf _{y \in \Lambda_{1}} I(y)<\inf _{y \in \partial \Lambda_{1}} I(y)
$$

in particular, there exists $\underline{y} \in \Lambda_{1}$ such that $I(\underline{y})=\inf _{y \in \Lambda_{1}} I(y)$.

Proof. - Fix $T>0$, let $I_{T}$ be the corresponding functional and let $\underline{y}^{T} \in \bar{\Lambda}_{1}$ be the minimum of $I_{T}$ over $\bar{\Lambda}_{1}$ obtained in Lemma 5: we claim that $y^{T} \notin \partial \Lambda_{1}$ for $T$ and $m_{3}$ sufficiently small. By contradiction, let $y \in \partial \Lambda_{1}^{-}$be a collision minimum, that is, $I(y)=\inf _{\Lambda_{1}} I$, where we have set $I=I_{T}$. By Lemma 6 we know that $\left(y_{1}, y_{2}\right)$ is close in the norm topology of $H^{1}\left([0,1], \mathbb{R}^{4}\right)$ to some point in $\Sigma$; in particular, $y_{1}(t) \neq y_{2}(t)$ for all $t \in[0,1]$. Moreover, if we set

$$
V(q)=-\frac{m_{1}}{\left|q-y_{1}\right|}-\frac{m_{2}}{\left|q-y_{2}\right|},
$$


then $y_{3}$ minimizes the restricted functional

$$
\Psi(q)=\int_{0}^{1} \frac{1}{2}|\dot{q}+T \vartheta J q|^{2}-T^{2} V(q)
$$

on the set $\Omega$ of functions $q \in H^{1}\left([0,1], \mathbb{R}^{2}\right)$ satisfying $q(0)=q(1)$, $q(t) \neq y_{i}(t)$ for all $t \in[0,1]$ and $i=1,2$ and $\operatorname{ind}\left(q-y_{1}\right)=-1$.

We set $e(t)=T^{-2 / 3} y_{1}(t), q(x)=T^{2 / 3}(x+e)$ and $\bar{\Phi}(x)=T^{-4 / 3} \Psi$ $(q(x))$; then, we infer that $y_{0}(t)=T^{-2 / 3}\left(y_{3}(t)-y_{1}(t)\right)$ minimizes the functional

$$
\begin{aligned}
\bar{\Phi}(x)= & \int_{0}^{1}\left[\frac{1}{2}|\dot{x}|^{2}+\frac{m_{1}}{|x|}+(\dot{x}, \dot{e})+T \vartheta(\dot{x}, J x)+2 T \vartheta(\dot{e}, J x)\right. \\
& \left.+\frac{T^{2} \vartheta^{2}}{2}|x|^{2}+T^{2} \vartheta^{2}(e, x)+T^{2 / 3} \frac{m_{2}}{\left|T^{2 / 3}(x+e)-y_{2}\right|}\right]+c(e),
\end{aligned}
$$

where

$$
c(e)=\int_{0}^{1} \frac{|\dot{e}|^{2}}{2}+T \vartheta(\dot{e}, J e)+\frac{T^{2} \vartheta^{2}}{2}|e|^{2} .
$$

Next we define

$$
\Phi(x)=\bar{\Phi}(x)-c(e)-\int_{0}^{1} \frac{m_{2} T^{2 / 3}}{\left|y_{2}\right|},
$$

so that $y_{0}$ also minimizes the functional

$$
\begin{aligned}
\Phi(x)= & \int_{0}^{1}\left[\frac{1}{2}|\dot{x}|^{2}+\frac{m_{1}}{|x|}+(\dot{x}, \dot{e})+T \vartheta(\dot{x}, J x)+2 T \vartheta(\dot{e}, J x)\right. \\
& \left.+\frac{T^{2} \vartheta^{2}}{2}|x|^{2}+T^{2} \vartheta^{2}(e, x)+T^{4 / 3} m_{2} G(x)\right],
\end{aligned}
$$

where $G(x)$ is the smooth function given by

$$
G(x)=T^{-2 / 3}\left(\frac{1}{\left|T^{2 / 3}(x+e)-y_{2}\right|}-\frac{1}{\left|y_{2}\right|}\right)
$$


moreover, since $y_{2}(t) \neq 0$ for all $t$, by the asymptotic expansion

$$
\frac{1}{|\varepsilon u-v|}=\frac{1}{|v|}\left(1+\varepsilon \frac{(u, v)}{|v|}+\mathrm{o}(\varepsilon)\right)
$$

(which holds for all $u, v \in \mathbb{R}^{2} \backslash\{0\}$ as $\varepsilon \rightarrow 0$ ) we have as $T \rightarrow 0$

$$
G(x)=\left(x+e, \frac{y_{2}}{\left|y_{2}\right|^{3}}\right)+\mathrm{o}(1) .
$$

Let $\Xi=\left\{t \in[0,1]: y_{0}(t)=0\right\}$. It is well known [2] that $\Xi$ has measure zero and that $y_{0}(t)$ satisfies the Euler equation corresponding to $\Phi$ for all $t \notin \Xi$, namely

$$
\begin{aligned}
\ddot{y}_{0}= & -2 T \vartheta J \dot{y}_{0}-T^{2} \vartheta^{2} y_{0}+T^{2} \vartheta^{2} e-2 T \vartheta J \dot{e}-\ddot{e}-m_{1} \frac{y_{0}}{\left|y_{0}\right|^{3}} \\
& +\left(T^{4 / 3} m_{2}\right) \nabla G\left(y_{0}\right)
\end{aligned}
$$

and if we compute the scalar product of this equation by $J y_{0}$ we obtain

$$
\begin{aligned}
\frac{\mathrm{d}}{\mathrm{d} t}\left(\dot{y}_{0}, J y_{0}\right)=\left(\ddot{y}_{0}, J y_{0}\right)= & \left(-2 T \vartheta J \dot{y}_{0}+T^{2} \vartheta^{2} e-2 T \vartheta J \dot{e}-\ddot{e}, J y_{0}\right) \\
& +T^{4 / 3} m_{2}\left(\nabla G\left(y_{0}\right), J y_{0}\right) ;
\end{aligned}
$$

since $y_{0} \in \partial \Lambda_{1}$ we may assume that 0 is a collision time, i.e. $\left(\dot{y}_{0}, J y_{0}\right)(0)$ $=0$ : hence, by integrating the previous equation on the interval $[0, t]$ with $0<t \leqslant 1$, we get

$$
\begin{aligned}
\left(\dot{y}_{0}, J y_{0}\right)(t)= & -2 T \vartheta \int_{0}^{t}\left(\dot{y}_{0}, y_{0}\right)+T^{2} \vartheta^{2} \int_{0}^{t}\left(e, J y_{0}\right)-2 T \vartheta \int_{0}^{t}\left(\dot{e}, y_{0}\right) \\
& +\int_{0}^{t}\left(\dot{e}, J \dot{y}_{0}\right)+T^{4 / 3} m_{2} \int_{0}^{t}\left(\nabla G\left(y_{0}\right), J y_{0}\right) .
\end{aligned}
$$

Now we estimate the integrals in (4.7): since, $y_{0}=y_{0}(T)$ is bounded in $H^{1}$ as $T \rightarrow 0$, we have

$$
\left|2 T \vartheta \int_{0}^{t}\left(\dot{y}_{0}, y_{0}\right)\right| \leqslant 2 T \vartheta\left\|\dot{y}_{0}\right\|_{2}\left\|y_{0}\right\|_{2} \leqslant 2 \lambda T
$$

for a.e. $t \in[0,1]$. Choose a constant $\mu>0$ and let

$$
m_{3} \leqslant \mu T^{8 / 3}
$$


since $e(t)=T^{-2 / 3} y_{1}(t)$, by Lemma 6 we have $\|\dot{e}\|_{2} \leqslant \mu T^{4 / 3}$ and therefore

$$
T^{2} \vartheta^{2} \int_{0}^{t}\left(e, J y_{0}\right)-2 T \vartheta \int_{0}^{t}\left(\dot{e}, y_{0}\right)+\int_{0}^{t}\left(\dot{e}, J \dot{y}_{0}\right)=\mathrm{o}(T)
$$

for a.e. $t \in[0,1]$. Hence, by (4.7), (4.8) and (4.10) we obtain for small $T$

$$
\left\|\left(\dot{y}_{0}, J y_{0}\right)\right\|_{\infty} \leqslant \lambda T
$$

Consider the function

$$
X(t)=R(\sin 2 \pi t, \cos 2 \pi t), \quad \text { where } R=\sqrt[3]{m_{1} /\left(4 \pi^{2}\right)},
$$

which minimizes the functional

$$
\hat{I}(x)=\int_{0}^{1} \frac{|\dot{x}|^{2}}{2}+\frac{m_{1}}{|x|}
$$

under the constraint ind $(x)=-1$; we have $(\dot{X}, J X) \equiv-2 \pi R^{2}$ and therefore

$$
\begin{aligned}
& \Phi(X)-\Phi\left(y_{0}\right) \\
& =\hat{I}(X)-\hat{I}\left(y_{0}\right)+T \vartheta \int_{0}^{1}\left[(\dot{X}, J X)-\left(\dot{y}_{0}, J y_{0}\right)\right]+\mathrm{o}(T)<0
\end{aligned}
$$

for small $T$. This contradicts the assumption that $y_{0}$ minimizes $\Phi$ and proves the lemma.

To complete the proof of Theorem 3, note that a noncollision critical point $x=\left(x_{1}, x_{2}, x_{3}\right)$ of $\Phi$ satisfies (2.4); then, it also satisfies

$$
\left(\dot{x}_{i}(T), p(T)\right)=\left(\dot{x}_{i}(0), p(0)\right)=\left(R_{\vartheta T} \dot{x}_{i}(0), p(T)\right), \quad i=1,2,3,
$$

for all $p \in H^{1}\left([0, T], \mathbb{R}^{2}\right)$; hence, $\dot{x}_{i}(T)=R_{\vartheta T} \dot{x}_{i}(0)$. This proves that the motion $\underline{x}$ is periodic if $\theta T / \pi \in \mathbb{Q}$ while it is quasi-periodic if $\theta T / \pi \in \mathbb{R} \backslash \mathbb{Q}$. 


\section{THE 4-BODY PROBLEM}

In this section we prove Theorem 4.

We may assume that $m_{4} \leqslant m_{3}$. As in the previous section, by rotating and rescaling, the Lagrangian becomes

$$
I(y)=\int_{0}^{1} \sum_{i=1}^{4} \frac{m_{i}}{2}\left|\dot{y}_{i}+T \vartheta J y_{i}\right|^{2}-T^{2} V(y),
$$

where

$$
V(y)=-\sum_{1 \leqslant i<j \leqslant 4} \frac{G m_{i} m_{j}}{\left|y_{j}-y_{i}\right|}
$$

we consider the Hilbert space $H$ defined by

$$
\begin{aligned}
& H=\left\{y=\left(y_{1}, y_{2}, y_{3}, y_{4}\right) \in H^{1}\left([0,1], \mathbb{R}^{8}\right): y(1)=y(0),\right. \\
& \left.\sum_{i=1}^{4} m_{i} y_{i}(t) \equiv 0\right\}
\end{aligned}
$$

the corresponding noncollision open set $\Lambda_{0}$ and its subset $\Lambda_{1}=\{y \in$ $\Lambda_{0}$ : ind $\left.\left(y_{i}-y_{1}\right)=-1, i=3,4\right\}$. By arguing as in Lemmas 4 and 5 one can prove that the Lagrangian achieves a minimum on $\bar{\Lambda}_{1}$ :

LEMmA 8. - There exists $\underline{y} \in \bar{\Lambda}_{1}$ such that $I(\underline{y})=\inf _{y \in \Lambda_{1}} I(y)$.

We can exclude the collisions between $B_{2}$ and $B_{3}$ and the collisions between $B_{2}$ and $B_{4}$.

Let $I_{0}$ be the functional corresponding to the case $m_{3}=m_{4}=0$, let $H_{0}$ and $\Sigma$ be as in the previous section and consider again the problem (4.2); then we obtain

LEMmA 9. - There exist two constants $\bar{T}, M>0$ depending only on $\theta, m_{1}, m_{2}$ and a constant $c>0$ such that for all $T \leqslant \bar{T}$, all $m_{4} \leqslant m_{3} \leqslant M$ and all $y=\left(y_{1}, y_{2}, y_{3}, y_{4}\right) \in H$ achieving the minimum in (4.1) (as given by Lemma 8) the following holds

$$
\sum_{i=1}^{2}\left(\left\|\dot{y}_{i}\right\|_{2}^{2}+T^{2}\left\|y_{i}-y_{i}^{\Sigma}\right\|_{2}^{2}\right) \leqslant c T^{4 / 3} m_{3},
$$

where $y_{i}^{\Sigma}=y_{i}^{\Sigma}(t)$ is the (pointwise) projection of $y_{i}(t)$ onto $\Sigma$. 
Proof. - Take any $\left(y_{E}, y_{S}\right) \in \Sigma$ and consider the function $Y \in H$ defined by

$$
\left\{\begin{array}{l}
Y_{1}(t) \equiv y_{E} \\
Y_{2}(t) \equiv y_{S} \\
Y_{3}(t)=y_{E}+T^{2 / 3}(\sin 2 \pi t, \cos 2 \pi t) \\
Y_{4}(t)=y_{E}-T^{2 / 3}(\sin 2 \pi t, \cos 2 \pi t),
\end{array}\right.
$$

so that (4.4) holds (recall that $m_{4} \leqslant m_{3}$ ). The proof may now be completed as in Lemma 6.

In particular, Lemma 9 excludes collisions between $B_{1}$ and $B_{2}$.

Now we return to the original problem: in order to prove Theorem 4 it suffices to show that the functional

$$
\Phi(x)=\int_{0}^{T} \sum_{i=1}^{4} \frac{m_{i}}{2}\left|\dot{x}_{i}\right|^{2}+\sum_{1 \leqslant i<j \leqslant 4} \frac{G m_{i} m_{j}}{\left|x_{j}-x_{i}\right|}
$$

satisfies

$$
\inf _{\Lambda_{1}} \Phi<\inf _{\partial \Lambda_{1}} \Phi
$$

indeed, by Lemma 8 , this would imply that $\Phi$ achieves a minimum over $\Lambda_{1}$. Here $\Phi$ is defined on the space

$$
H=\left\{x=\left(x_{1}, x_{2}, x_{3}, x_{4}\right), x_{i} \in H^{1}\left([0, T], \mathbb{R}^{2}\right): x_{i}(T)=R_{\vartheta T} x_{i}(0)\right\}
$$

and $\Lambda_{1}$ is given by

$$
\Lambda_{1}=\left\{x \in \Lambda_{0}, \text { ind }\left(x_{i}-x_{1}\right)=-1, i=3,4\right\},
$$

where $\Lambda_{0}=\left\{x \in H, x_{i}(t) \neq x_{j}(t) \forall t \in[0, T] \forall i \neq j\right\}$.

From now on we denote by $\underline{x}=\left(\underline{x}_{1}, \underline{x}_{2}, \underline{x}_{3}, \underline{x}_{4}\right)$ the minimum of $\Phi$ over $\bar{\Lambda}_{1}$ corresponding to the orbit $y$ obtained in Lemma 8; we exclude the collisions of the two satellites with $B_{1}$.

LEMMA 10. - If T, $m_{3}\left(\right.$ and $\left.m_{4}\right)$ are sufficiently small, then $\underline{x}_{3}(t) \neq$ $\underline{x}_{1}(t)$ and $\underline{x}_{4}(t) \neq \underline{x}_{1}(t)$ for all $t \in[0, T]$.

Proof. - Consider the functional

$$
F(x)=\int_{0}^{T} \frac{|\dot{x}|^{2}}{2}+\frac{G m_{1}}{\left|x-\underline{x}_{1}\right|}+\frac{G m_{2}}{\left|x-\underline{x}_{2}\right|}
$$


defined on the space $K_{1}=\left\{x \in H^{1}\left([0, T], \mathbb{R}^{2}\right)\right\}$ and the corresponding noncollision set

$$
\Omega_{1}=\left\{x \in K_{1}, x(t) \neq \underline{x}_{1}(t) \quad \forall t \in[0, T] \text {, ind }\left(x-\underline{x}_{1}\right)=-1\right\} .
$$

Consider also the functionals

$$
\bar{\Phi}\left(x_{3}, x_{4}\right)=\int_{0}^{T}\left(\sum_{i=3}^{4} \frac{m_{i}}{2}\left|\dot{x}_{i}\right|^{2}-V(x)\right),
$$

where

$$
V(x)=-\frac{G m_{1} m_{3}}{\left|x_{3}-\underline{x}_{1}\right|}-\frac{G m_{1} m_{4}}{\left|x_{4}-\underline{x}_{1}\right|}-\frac{G m_{2} m_{3}}{\left|x_{3}-\underline{x}_{2}\right|}-\frac{G m_{2} m_{4}}{\left|x_{4}-\underline{x}_{2}\right|}-\frac{G m_{3} m_{4}}{\left|x_{3}-x_{4}\right|}
$$

and

$$
\Psi\left(x_{3}, x_{4}\right)=\sum_{i=3}^{4} m_{i} F\left(x_{i}\right)=\bar{\Phi}\left(x_{3}, x_{4}\right)-\int_{0}^{T} \frac{G m_{3} m_{4}}{\left|x_{4}-x_{3}\right|}
$$

defined on the space $K_{2}=\left\{x=\left(x_{3}, x_{4}\right), x_{i} \in H^{1}\left([0, T], \mathbb{R}^{2}\right)\right\}$; finally, consider the noncollision set

$$
\Omega_{2}=\left\{x=\left(x_{3}, x_{4}\right) \in K_{2}, x_{3}, x_{4} \in \Omega_{1}, x_{3}(t) \neq x_{4}(t) \forall t \in[0, T]\right\} .
$$

Since $\underline{x}$ minimizes $\Phi$ over $\bar{\Lambda}_{1}$, the couple $\left(\underline{x}_{3}, \underline{x}_{4}\right)$ minimizes the functional $\bar{\Phi}$ over $\bar{\Omega}_{2}$.

Take $T>0$ sufficiently small and $m_{3} \leqslant \mu T^{8 / 3}$ as in (4.9); then, by Lemma 7, there exists $C_{T}>0$ (independent of $m_{3}$ and $m_{4}$ ) such that

$$
\inf _{x \in \partial \Omega_{1}} F(x)-\inf _{x \in \Omega_{1}} F(x) \geqslant C_{T},
$$

the two infima being in fact two minima: let $X$ be one minimum of $F$ over $\Omega_{1}$. By definition of $\Psi$ we also have

$$
\inf _{\partial \Omega_{2}} \Psi \geqslant \inf _{\Omega_{2}} \Psi+C_{T} m_{4}
$$

and the minimum of $\Psi$ over $\Omega_{2}$ is achieved by $(X(t), X(t+s))$ for any $s \in[0, T]$. Now take $X_{3}(t)=X(t)$ and take $X_{4}(t)=X(t+T / 2)$ so that $X_{4}$ is also a noncollision minimum of $F$. Since $X$ minimizes $F$, then a 
simple argument shows that $X_{3}(t) \neq X_{4}(t)$ for all $t$ and we can define $C_{0}=\int_{0}^{T} \frac{G}{\left|X_{3}-X_{4}\right|}$. Assume by contradiction that $\left(\underline{x}_{3}, \underline{x}_{4}\right) \in \partial \Omega_{2}$; then, by (5.1), we have

$$
\begin{aligned}
\bar{\Phi}\left(\underline{x}_{3}, \underline{x}_{4}\right) & =\Psi\left(\underline{x}_{3}, \underline{x}_{4}\right)+\int_{0}^{T} \frac{G m_{3} m_{4}}{\left|\underline{x}_{4}-\underline{x}_{3}\right|} \geqslant \inf _{\partial \Omega_{2}} \Psi+\int_{0}^{T} \frac{G m_{3} m_{4}}{\left|\underline{x}_{4}-\underline{x}_{3}\right|} \\
& \geqslant \bar{\Phi}\left(X_{3}, X_{4}\right)-\int_{0}^{T} \frac{G m_{3} m_{4}}{\left|X_{4}-X_{3}\right|}+\int_{0}^{T} \frac{G m_{3} m_{4}}{\left|\underline{x}_{4}-\underline{x}_{3}\right|}+C_{T} m_{4} \\
& \geqslant \bar{\Phi}\left(X_{3}, X_{4}\right)-C_{0} m_{3} m_{4}+C_{T} m_{4}
\end{aligned}
$$

and therefore $\bar{\Phi}\left(\underline{x}_{3}, \underline{x}_{4}\right)>\bar{\Phi}\left(X_{3}, X_{4}\right)$ for sufficiently small $m_{3}$ (and $m_{4}$ ): this contradicts the assumption that $\left(\underline{x}_{3}, \underline{x}_{4}\right)$ minimizes $\bar{\Phi}$ over $\bar{\Omega}_{2}$ and proves the lemma.

Finally, we exclude the case where the two satellites collide with each other:

LEMMA $11 .-\underline{x}_{3}(t) \neq \underline{x}_{4}(t)$ for all $t \in[0, T]$.

Proof. - We make the following change of variables: let

$$
\left\{\begin{array}{l}
X=\frac{m_{3} x_{3}+m_{4} x_{4}}{m_{3}+m_{4}} \\
r=x_{4}-x_{3}
\end{array}\right.
$$

and we denote by $(\underline{X}, \underline{r})$ the couple corresponding to $\left(\underline{x}_{3}, \underline{x}_{4}\right)$. We focus our attention on $x_{3}$ and $x_{4}$ and we consider the restricted Lagrangian

$$
\Psi(X, r)=\int_{0}^{T} \frac{m_{3}+m_{4}}{2}|\dot{X}|^{2}+\frac{m_{3} m_{4}}{2\left(m_{3}+m_{4}\right)}|\dot{r}|^{2}+\frac{G m_{3} m_{4}}{|r|}-V_{R}(X, r),
$$

where

$$
V_{R}(X, r)=-\frac{G m_{1} m_{3}}{\left|x_{3}-\underline{x}_{1}\right|}-\frac{G m_{1} m_{4}}{\left|x_{4}-\underline{x}_{1}\right|}-\frac{G m_{2} m_{3}}{\left|x_{3}-\underline{x}_{2}\right|}-\frac{G m_{2} m_{4}}{\left|x_{4}-\underline{x}_{2}\right|} .
$$

Obviously,

$$
\Phi\left(\underline{x}_{1}, \underline{x}_{2}, x_{3}, x_{4}\right)=\Psi(X, r)+\int_{0}^{T} \frac{m_{1}}{2}\left|\underline{\dot{x}}_{1}\right|^{2}+\frac{m_{2}}{2}\left|\underline{\dot{x}}_{2}\right|^{2}+\frac{G m_{1} m_{2}}{\left|\underline{x}_{2}-\underline{x}_{1}\right|} .
$$


By contradiction, assume that in the motion described by $\underline{x}=\underline{x}(t)$ the satellites $B_{3}$ and $B_{4}$ collide with each other, but not with $B_{1}$, at $t=0$, that is $\underline{r}(0)=0$ and $\underline{X}(0) \neq \underline{x}_{1}(0)$. Let $\varepsilon>0$ satisfy

$$
\varepsilon<\frac{1}{2} \min \left\{\left|\underline{X}(0)-\underline{x}_{1}(0)\right|,\left|\underline{x}_{3}(0)-\underline{x}_{2}(0)\right|,\left|\underline{x}_{4}(0)-\underline{x}_{2}(0)\right|\right\}
$$

and let $t_{\varepsilon}<0<t^{\varepsilon}$ be such that $\left|\underline{r}\left(t_{\varepsilon}\right)\right|=\left|\underline{r}\left(t^{\varepsilon}\right)\right|=\varepsilon$ and $|\underline{r}(t)|<\varepsilon$ if $t \in\left(t_{\varepsilon}, t^{\varepsilon}\right)$; if $\varepsilon$ is sufficiently small (say $\varepsilon<\bar{\varepsilon}$ ), then $\underline{r}(t) \neq 0$ for all $t \in\left(t_{\varepsilon}, t^{\varepsilon}\right) \backslash\{0\}$. We will get a contradiction by showing that there exists $\varepsilon \in(0, \bar{\varepsilon})$ and $\hat{r}:\left[t_{\varepsilon}, t^{\varepsilon}\right] \rightarrow \mathbb{R}^{2} \backslash\{0\}$ such that the function

$$
R(t)= \begin{cases}\underline{r}(t) & \text { if } t \notin\left[t_{\varepsilon}, t^{\varepsilon}\right], \\ \hat{r}(t) & \text { if } t \in\left[t_{\varepsilon}, t^{\varepsilon}\right],\end{cases}
$$

satisfies $R \in H^{1}\left([0, T], \mathbb{R}^{2}\right)$ and $\Psi(\underline{X}, \underline{r})>\Psi(\underline{X}, R)$.

We may assume that $\underline{r}\left(t_{\varepsilon}\right)=(\varepsilon, 0)$ and $\underline{r}\left(t^{\varepsilon}\right)=\varepsilon \mathrm{e}^{i \alpha}$ for some $\alpha \in$ $(-\pi, \pi]$; let $p=\varepsilon^{3 / 2} \mathrm{e}^{i \alpha / 2}$. Let

$$
\ell=\frac{\left|\underline{r}\left(t_{\varepsilon}\right)-p\right|}{\varepsilon}=\frac{\left|\underline{r}\left(t^{\varepsilon}\right)-p\right|}{\varepsilon} ;
$$

since $|\alpha| / 2 \leqslant \pi / 2$, we have

$$
\ell^{2}=\frac{\left|\underline{r}\left(t_{\varepsilon}\right)\right|^{2}+|p|^{2}-2\left(\underline{r}\left(t_{\varepsilon}\right), p\right)}{\varepsilon^{2}}=\frac{\varepsilon^{2}+\varepsilon^{3}-2\left(\underline{r}\left(t_{\varepsilon}\right), p\right)}{\varepsilon^{2}} \leqslant 1+\varepsilon .
$$

Let $S_{1}$ and $S_{2}$ be respectively the segments connecting $\underline{r}\left(t_{\varepsilon}\right)$ and $\underline{r}\left(t^{\varepsilon}\right)$ with $p$ and let $\hat{r}(t)$ be such that

$$
\begin{array}{llll}
\hat{r}(t) \in S_{1} \quad \text { and } & |\hat{r}(t)-p|=\ell|\underline{r}(t)| & \forall t \in\left[t_{\varepsilon}, 0\right], \\
\hat{r}(t) \in S_{2} \quad \text { and } & |\hat{r}(t)-p|=\ell|\underline{r}(t)| & \forall t \in\left[0, t^{\varepsilon}\right] .
\end{array}
$$

Since the motion of $\hat{r}$ is straight, for all $t \in\left[t_{\varepsilon}, 0\right]$ we get

$$
\begin{aligned}
|\hat{r}(t)-p| \cdot\left|\frac{\mathrm{d}}{\mathrm{d} t} \hat{r}(t)\right| & =\left|\left(\hat{r}(t)-p, \frac{\mathrm{d}}{\mathrm{d} t}(\hat{r}(t)-p)\right)\right| \\
& =\left|\frac{1}{2} \frac{\mathrm{d}}{\mathrm{d} t}\right| \hat{r}(t)-\left.p\right|^{2}\left|=\frac{\ell^{2}}{2}\right| \frac{\mathrm{d}}{\mathrm{d} t}|\underline{r}(t)|^{2} \mid \\
& \leqslant \ell^{2}|\underline{r}(t)| \cdot\left|\frac{\mathrm{d}}{\mathrm{d} t} \underline{r}(t)\right|,
\end{aligned}
$$


and by (5.2)

$$
\left|\frac{\mathrm{d}}{\mathrm{d} t} \hat{r}(t)\right|^{2} \leqslant \ell^{2}\left|\frac{\mathrm{d}}{\mathrm{d} t} \underline{r}(t)\right|^{2} \leqslant(1+\varepsilon)\left|\frac{\mathrm{d}}{\mathrm{d} t} \underline{r}(t)\right|^{2}
$$

similarly, we obtain the same inequality when $t \in\left[0, t^{\varepsilon}\right]$. Next, note that

$$
V_{R}(\underline{X}(t), \hat{r}(t))-V_{R}(\underline{X}(t), \underline{r}(t)) \geqslant-c \varepsilon \quad \text { for all } t \in\left[t_{\varepsilon}, t^{\varepsilon}\right]
$$

this, together with (5.3) yields

$$
\begin{aligned}
& \int_{t_{\varepsilon}}^{t^{\varepsilon}} \frac{m_{3} m_{4}}{2\left(m_{3}+m_{4}\right)}\left(\left|\frac{\mathrm{d} \underline{r}}{\mathrm{~d} t}\right|^{2}-\left|\frac{\mathrm{d} \hat{r}}{\mathrm{~d} t}\right|^{2}\right) \\
& \quad+V_{R}(\underline{X}(t), \hat{r}(t))-V_{R}(\underline{X}(t), \underline{r}(t)) \geqslant-c \varepsilon .
\end{aligned}
$$

To estimate $\frac{1}{|\underline{r}|}-\frac{1}{|\hat{r}|}$, we argue as in [17]: by conservation of the total energy $E$ we obtain for all $t \in\left[t_{\varepsilon}, t^{\varepsilon}\right]$

$$
\begin{aligned}
c|\underline{\dot{r}}(t)|^{2} \leqslant & \frac{m_{1}}{2}\left|\underline{\dot{x}}_{1}(t)\right|^{2}+\frac{m_{2}}{2}\left|\underline{\dot{x}}_{2}(t)\right|^{2}+\frac{m_{3}}{2}\left|\underline{\dot{x}}_{3}(t)\right|^{2}+\frac{m_{4}}{2}\left|\underline{\dot{x}}_{4}(t)\right|^{2} \\
= & E+\frac{G m_{1} m_{2}}{\left|\underline{x}_{2}(t)-\underline{x}_{1}(t)\right|}+\frac{G m_{1} m_{3}}{\left|\underline{x}_{3}(t)-\underline{x}_{1}(t)\right|}+\frac{G m_{1} m_{4}}{\left|\underline{x}_{4}(t)-\underline{x}_{1}(t)\right|} \\
& +\frac{G m_{2} m_{3}}{\left|\underline{x}_{3}(t)-\underline{x}_{2}(t)\right|}+\frac{G m_{2} m_{4}}{\left|\underline{x}_{4}(t)-\underline{x}_{2}(t)\right|}+\frac{G m_{3} m_{4}}{|\underline{r}(t)|} \leqslant \frac{c}{|\underline{r}(t)|},
\end{aligned}
$$

the latter inequality being consequence of the fact that there exists $K>0$ such that for all $\varepsilon \in(0, \bar{\varepsilon})$ we have

$$
\inf _{t \in\left(t_{\varepsilon}, t^{\varepsilon}\right)} \min \left\{\left|\underline{x}_{j}(t)-\underline{x}_{i}(t)\right|, 1 \leqslant i<j \leqslant 4,(i, j) \neq(3,4)\right\} \geqslant K .
$$

Therefore, if we let $\rho(t)=|\underline{r}(t)|$, we have

$$
\rho \dot{\rho}=\frac{1}{2} \frac{\mathrm{d}}{\mathrm{d} t} \rho^{2}=(\underline{r}(t), \underline{\dot{r}}(t)) \leqslant|\underline{r}(t)| \cdot|\underline{\dot{r}}(t)| \leqslant \rho \frac{c}{\sqrt{\rho}} ;
$$

this proves that

$$
\rho(t) \leqslant c t^{2 / 3} \quad \forall t \in\left[t_{\varepsilon}, t^{\varepsilon}\right] .
$$

Let $t_{\varepsilon}<s_{1}<0<s_{2}<t^{\varepsilon}$ satisfy $\left|\underline{r}\left(s_{i}\right)\right|=\varepsilon^{3 / 2} / 2$ and $|\underline{r}(t)|<\varepsilon^{3 / 2} / 2$ for all $t \in\left(s_{1}, s_{2}\right)$; by (5.5) we obtain $\left|s_{i}\right| \geqslant c \varepsilon^{9 / 4}$. Moreover, since 
$|\hat{r}(t)|>|\underline{r}(t)|$ for all $t \in\left(t_{\varepsilon}, t^{\varepsilon}\right)$ we infer that

$$
\int_{t_{\varepsilon}}^{s_{1}} \frac{1}{|\underline{r}|}>\int_{t_{\varepsilon}}^{s_{1}} \frac{1}{|\hat{r}|} \text { and } \int_{s_{2}}^{t^{\varepsilon}} \frac{1}{|\underline{r}|}>\int_{s_{2}}^{t^{\varepsilon}} \frac{1}{|\hat{r}|} ;
$$

on the other hand, $|\hat{r}(t)| \geqslant \varepsilon^{3 / 2} \geqslant 2|\underline{r}(t)|$ for all $t \in\left(s_{1}, s_{2}\right)$ so that, by (5.5), we obtain

$$
\begin{aligned}
G m_{3} m_{4} \int_{t_{\varepsilon}}^{t^{\varepsilon}} \frac{1}{|\underline{r}|}-\frac{1}{|\hat{r}|} & >G m_{3} m_{4} \int_{s_{1}}^{s_{2}} \frac{1}{|\underline{r}|}-\frac{1}{|\hat{r}|} \geqslant c \int_{-c \varepsilon^{9 / 4}}^{c \varepsilon^{9 / 4}} \frac{1}{2|\underline{r}|} \\
& \geqslant c \int_{-c \varepsilon^{9 / 4}}^{c \varepsilon^{9 / 4}} \frac{1}{t^{2 / 3}} \geqslant c \varepsilon^{3 / 4} .
\end{aligned}
$$

This, together with (5.4), implies that $\Psi(\underline{X}, \underline{r})-\Psi(\underline{X}, R) \geqslant c \varepsilon^{3 / 4}-c \varepsilon>$ 0 if $\varepsilon$ is small.

The proof of Theorem 4 may now be completed as in the previous section.

\section{NUMERICAL RESULTS}

In this section we prove Theorem 2 and we give some numerical results illustrating the orbits determined in Theorem 1. Since all the solutions we obtained are minima, it is possible to numerically compute them by a rather simple procedure. The technique is standard: one chooses a finite dimensional vector space $H_{f}$ which approximates the Hilbert space $H$, introduces a functional $\Phi_{f}: H_{f} \rightarrow \mathbb{R}$ approximating $\Phi$ and looks for minima of $\Phi_{f}$ by choosing an arbitrary starting point $x_{0} \in H_{f}$ and defining a sequence $\left\{x_{n}\right\}$ by setting $x_{n+1}=x_{n}-$ $h_{n} \nabla \Phi_{f}\left(x_{n}\right)$, where $\nabla \Phi_{f}: H_{f} \rightarrow H_{f}$ is the gradient of $\Phi$ and represents the maximum slope direction of the functional $\Phi_{f}$, while $h_{n}$ is computed at each step in order to minimize the function $h \mapsto \Phi\left(x_{n+1}(h)\right)$. If the approximated functional maintains the properties of $\Phi$, then the sequence $\left\{x_{n}\right\}$ converges to a minimum point of the functional $\Phi_{f}$.

We only treat the restricted problem (although there are no obstructions to the treatment of the complete problem). As an approximate space $H_{f}$ we chose the set of closed $m$-gonals and we let $m$ vary between 100 and 300 , depending on the values of the parameters. A function $x \in H_{f}$ is 
uniquely characterized by the coordinates of the vertices, therefore it can be represented by a point in $\mathbb{R}^{2 m}$. Given $x \in H_{f}$ by $x_{i} \in \mathbb{R}^{2}$ we denote the coordinates of the $i$ th vertex. The (full) functional we consider is

$$
\begin{aligned}
\Phi(x)= & \int_{0}^{1} \frac{1}{2}|\dot{x}+v J x|^{2}+\frac{1}{|x|}+\frac{\rho}{\left|x-(\rho+1)^{1 / 3} v^{-2 / 3} e_{1}\right|} \\
& -\frac{\rho v^{4 / 3}}{(\rho+1)^{2 / 3}}\left(x, e_{1}\right),
\end{aligned}
$$

and its representation in $H_{f}$ is given by

$$
\begin{aligned}
\Phi_{f}(x)= & \sum_{i=1}^{m} \frac{1}{2}\left|\frac{x_{i+1}-x_{i}}{h}+v J x_{i}\right|^{2}+\frac{1}{\left|x_{i}\right|}+\frac{\rho}{\left|x_{i}-(\rho+1)^{1 / 3} v^{-2 / 3} e_{1}\right|} \\
& -\frac{\rho v^{4 / 3}}{(\rho+1)^{2 / 3}}\left(x_{i}, e_{1}\right),
\end{aligned}
$$

where $h=1 / m$. We also have

$$
\begin{aligned}
\frac{\partial \Phi_{f}(x)}{\partial x_{i}}= & -\frac{x_{i+1}-2 x_{i}+x_{i-1}}{h^{2}}-\frac{x_{i}}{\left|x_{i}\right|^{3}}-2 v J \frac{x_{i+1}-x_{i}}{h}+v^{2} x_{i} \\
& -\rho \frac{x_{i}-(\rho+1)^{1 / 3} v^{-2 / 3} e_{1}}{\left|x_{i}-(\rho+1)^{1 / 3} v^{-2 / 3} e_{1}\right|^{3}}-\frac{\rho v^{4 / 3}}{(\rho+1)^{2 / 3}} e_{1} .
\end{aligned}
$$

Clearly, also in the numerical approximation we have to cope with the presence of the singularity in the potential; furthermore is it not very clear how to implement the topological constraint. In order to overcome these problems we introduced a naive method, i.e. we checked that at every step no vertices of $x_{n}$ were too close to the singularities. More precisely we checked that the minimum of the distances of the vertices from the singularity was larger than the maximum of the length of the sides of the $m$-gonal. In fact this condition was satisfied at all times during all our computations.

The following pictures represent the results we obtained for various values of the parameters $v$ and $\rho$. As a starting point $x_{0}$ we chose the orbit corresponding to the solution for the case $v=0$, that is the circular orbit of radius $R=(2 \pi)^{-2 / 3}$. Although analytically we could exclude collisions in the case $\rho=(3.3) 10^{5}$ only for values of $v$ smaller than 0.8 , numerically it clearly appears that the minima of the functional is very close to a circular orbit even for values of $v$ up to 3 . For larger values the orbit becomes more similar to an ellipse, but still it does not come 


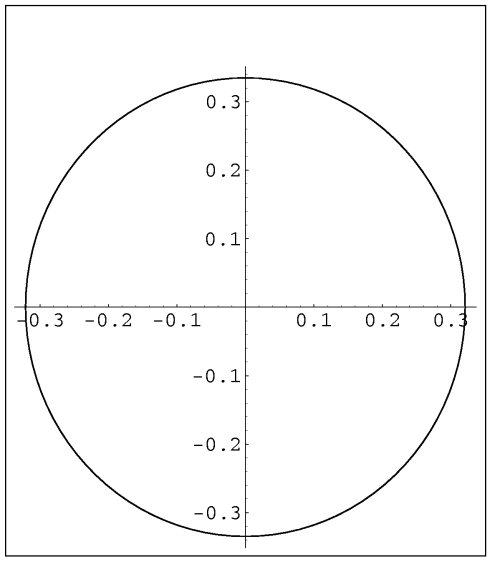

$\rho=330000, \nu=1$

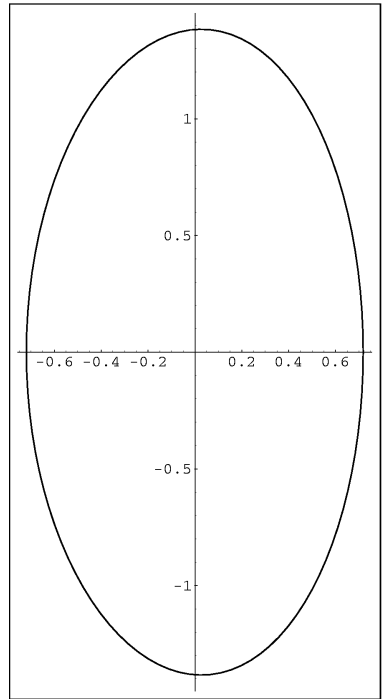

$\rho=330000 \nu=6$
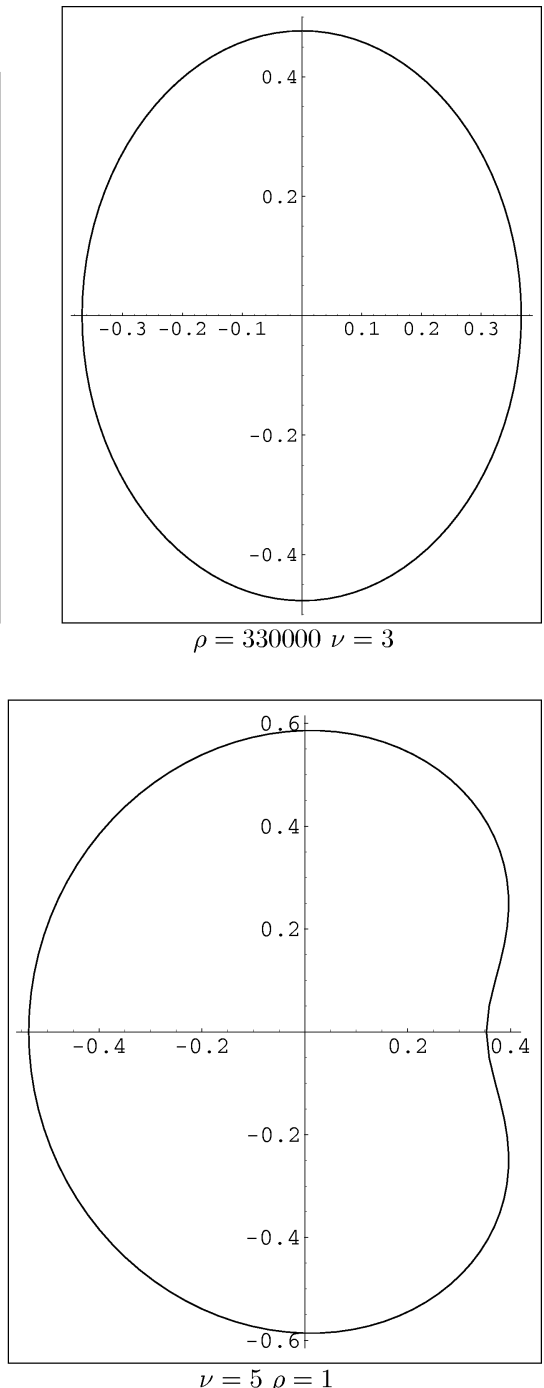

Fig. 1.

close to a collision orbit. In order to show some different behavior, we also show a picture (Fig. 1) in the case $\rho=1$ and $v=5$.

Proof of Theorem 2. - Let $\rho=(3.3) 10^{5}, v \leqslant 0.8$ and let $\underline{x}$ denote a corresponding orbit found in Lemma 1. We argue as in the proof of Lemma 3 making finer estimates. By contradiction, assume that $\underline{x} \in \partial \Lambda_{1}$ 
and consider again the function

$$
x_{0}=(2 \pi)^{-2 / 3}(\sin 2 \pi t, \cos 2 \pi t) .
$$

By arguing corresponding to (3.5) we find that (if $v \leqslant 0.8$ )

$$
\Phi\left(x_{0}\right)<\frac{3}{2}(2 \pi)^{2 / 3}-(0.541) v+(0.175) v^{2}<\frac{3}{2}(2 \pi)^{2 / 3} .
$$

Since $\underline{x}$ minimizes $\Phi$, by (3.3) and (6.1) we get

$$
\frac{1}{2}\left(1-\frac{v}{2 \pi}\right)^{2}\|\underline{\dot{x}}\|_{2}^{2}-\frac{\rho v^{4 / 3}}{\pi(\rho+1)^{2 / 3}}\|\underline{\dot{x}}\|_{2}-\frac{\rho v^{2 / 3}}{(\rho+1)^{1 / 3}} \leqslant \frac{3}{2}(2 \pi)^{2 / 3}
$$

by (3.4) this proves that

$$
\|\underline{\dot{x}}\|_{2}<130, \quad\|\underline{x}\|_{\infty}<65<(0.811) \frac{(\rho+1)^{1 / 3}}{v^{2 / 3}} .
$$

Now we claim that for all $t \in[0,1]$ we have

$$
\begin{aligned}
h(\underline{x}(t)) & :=\frac{\rho}{\left|\underline{x}(t)-(\rho+1)^{1 / 3} v^{-2 / 3} e_{1}\right|}-\frac{\rho v^{4 / 3}}{(\rho+1)^{2 / 3}}\left(\underline{x}(t), e_{1}\right) \\
& >-(0.035) \frac{\rho v^{2 / 3}}{(\rho+1)^{1 / 3}} .
\end{aligned}
$$

If $\left(\underline{x}(t), e_{1}\right) \leqslant 0,(6.4)$ follows readily. If $\left(\underline{x}(t), e_{1}\right)>0$, then by (6.3) we get

$$
\left|\underline{x}(t)-(\rho+1)^{1 / 3} v^{-2 / 3} e_{1}\right|^{2}<\|\underline{x}\|_{\infty}^{2}+\frac{(\rho+1)^{2 / 3}}{v^{4 / 3}}<(1.658) \frac{(\rho+1)^{2 / 3}}{v^{4 / 3}}
$$

then (6.4) follows by estimating $\left(\underline{x}(t), e_{1}\right)$ with $\|\underline{x}\|_{\infty}$ and by (6.3).

Since $v \leqslant 0.8$, by (6.4) we may replace (6.2) with

$$
(0.3807)\|\underline{\dot{x}}\|_{2}^{2}-(1.035) \frac{\rho v^{2 / 3}}{(\rho+1)^{1 / 3}} \leqslant \frac{3}{2}(2 \pi)^{2 / 3},
$$

which, by (3.4), proves that

$$
\|\underline{\dot{x}}\|_{2}<106, \quad\|\underline{x}\|_{\infty}<53<(0.661) \frac{(\rho+1)^{1 / 3}}{v^{2 / 3}} ;
$$


this allows to prove that

$$
h(\underline{x}(t))>(0.173) \frac{\rho v^{2 / 3}}{(\rho+1)^{1 / 3}} \quad \forall t \in[0,1] .
$$

Indeed, if $\left(\underline{x}(t), e_{1}\right) \leqslant 0$, we have

$$
h(\underline{x}(t)) \geqslant \frac{\rho}{\|\underline{x}\|_{\infty}+(\rho+1)^{1 / 3} v^{-2 / 3}}>(0.6) \frac{\rho v^{2 / 3}}{(\rho+1)^{1 / 3}} .
$$

If $\left(\underline{x}(t), e_{1}\right)>0$, then by arguing as for (6.4) and by taking into account (6.6) we get (6.7).

By (6.7) the inequality (6.5) becomes

$$
(0.3807)\|\underline{\dot{x}}\|_{2}^{2}-(0.827) \frac{\rho v^{2 / 3}}{(\rho+1)^{1 / 3}} \leqslant \frac{3}{2}(2 \pi)^{2 / 3},
$$

which, by (3.4), proves that

$$
\|\underline{\dot{x}}\|_{2}<94.8, \quad\|\underline{x}\|_{\infty}<47.4<(0.592) \frac{(\rho+1)^{1 / 3}}{v^{2 / 3}} .
$$

Finally, repeating once more the whole procedure, we get $h(\underline{x}(t))>$ $(0.267) \rho v^{2 / 3}(\rho+1)^{-1 / 3}$ which yields

$$
\|\underline{\dot{x}}\|_{2}<89.1, \quad\|\underline{x}\|_{\infty}<44.55<(0.556) \frac{(\rho+1)^{1 / 3}}{v^{2 / 3}} .
$$

Hence, $\left|s \underline{x}(t)-(\rho+1)^{1 / 3} v^{-2 / 3} e_{1}\right|>(0.444)(\rho+1)^{1 / 3} v^{-2 / 3}$ and

$$
\int_{0}^{1} \frac{\rho|\underline{x}|^{2}}{2\left|s \underline{x}-(\rho+1)^{1 / 3} v^{-2 / 3} e_{1}\right|^{3}}<\frac{v^{2}\|\underline{\dot{x}}\|_{2}^{2}}{2(0.444)^{3} \pi^{2}} \leqslant(0.3705)\|\underline{\dot{x}}\|_{2}^{2} .
$$

Therefore, by (3.1) and (6.1) we obtain

$$
\begin{aligned}
\frac{3}{2}(2 \pi)^{2 / 3} & >\Phi(\underline{x}) \geqslant(0.3807)\|\underline{\dot{x}}\|_{2}^{2}-\int_{0}^{1} \frac{\rho|\underline{x}|^{2}}{2\left|s \underline{x}-(\rho+1)^{1 / 3} v^{-2 / 3} e_{1}\right|^{3}} \\
& >(0.0102)\|\underline{\dot{x}}\|_{2}^{2} ;
\end{aligned}
$$


this proves that

$$
\|\underline{\dot{x}}\|_{2}<22.4, \quad\|\underline{x}\|_{\infty}<11.2<(0.14) \frac{(\rho+1)^{1 / 3}}{v^{2 / 3}} .
$$

Repeating this procedure we get

$$
\int_{0}^{1} \frac{\rho|\underline{x}|^{2}}{2\left|s \underline{x}-(\rho+1)^{1 / 3} v^{-2 / 3} e_{1}\right|^{3}}<\frac{v^{2}\|\underline{\dot{x}}\|_{2}^{2}}{2(0.86)^{3} \pi^{2}} \leqslant(0.051)\|\underline{\dot{x}}\|_{2}^{2} .
$$

Therefore, by (3.1), (6.1) and Jensen's inequality we obtain

$$
\frac{3}{2}(2 \pi)^{2 / 3}>(0.3297)\|\underline{\dot{x}}\|_{2}^{2}+\frac{\pi}{\|\underline{\dot{x}}\|_{2}},
$$

which proves that

$$
\|\underline{\dot{x}}\|_{2}<3.6, \quad\|\underline{x}\|_{\infty}<1.8<(0.023) \frac{(\rho+1)^{1 / 3}}{v^{2 / 3}}
$$

and

$$
\int_{0}^{1} \frac{\rho|\underline{x}|^{2}}{2\left|s \underline{x}-(\rho+1)^{1 / 3} v^{-2 / 3} e_{1}\right|^{3}}<\frac{v^{2}\|\underline{x}\|_{2}^{2}}{2(0.977)^{3}} \leqslant(0.537) v^{2}\|\underline{x}\|_{2}^{2} .
$$

Therefore, by arguing as in the proof of Lemma 3 we get

$$
\begin{aligned}
& \frac{3}{2}(2 \pi)^{2 / 3}-(0.541) v+(0.175) v^{2} \\
& >\Phi\left(x_{0}\right) \geqslant \Phi(\underline{x}) \geqslant \int_{0}^{1} \frac{1}{2}|\underline{\dot{x}}+v J \underline{x}|^{2}+\frac{1}{|\underline{x}|}-(0.537) v^{2}|\underline{x}|^{2} \\
& \geqslant\left(1-\frac{(1.074) v^{2}}{\pi^{2}}\right)^{1 / 3} \inf _{z \in H_{0}^{1}} \int_{0}^{1} \frac{1}{2}|\dot{z}|^{2}+\frac{1}{|z|} \\
& =\left(1-\frac{(1.074) v^{2}}{\pi^{2}}\right)^{1 / 3} \frac{3}{2}(2 \pi)^{2 / 3} .
\end{aligned}
$$

Since $v \leqslant 0.8$ we have $\left(1-\frac{(1.074) v^{2}}{\pi^{2}}\right)^{1 / 3} \geqslant 1-(0.038) v^{2}$ and therefore the last sequence of inequalities yields $(0.37) v>0.541$ which contradicts $v \leqslant 0.8$ and proves the statement. 


\section{REFERENCES}

[1] Albouy A., Chenciner A., Le problème des $n$ corps et les distances mutuelles, Invent. Math. 131 (1998) 151-184.

[2] Ambrosetti A., Critical points and nonlinear variational problems, Mémoire de la Société Mathématique de France 49, 1992.

[3] Ambrosetti A., Coti Zelati V., Periodic solutions of singular Lagrangian systems, Progress in Nonlinear Differential Equations and their Applications, Birkhäuser, 1993.

[4] Bessi U., Coti Zelati V., Symmetries and noncollision closed orbits for planar $\mathrm{N}$ body-type potentials, Nonlin. Anal. TMA 16 (1991) 587-598.

[5] Chenciner A., Desolneux N., Minima de l'intégrale d'action et équilibres relatifs de $n$ corps, C. R. Acad. Sci. Paris Ser. I Math. 326 (1998) 1209-1212 (Erratum: C. R. Acad. Sci. Paris Ser. I Math. 327 (1998) 193).

[6] Coti Zelati V., A class of periodic solutions of the $N$-body problem, Celestial Mech. Dynam. Astronom. 46 (2) (1989) 177-186.

[7] Coti Zelati V., Periodic solutions for $N$-body type problems, Ann. Inst. H. Poincaré Anal. Non Linéaire 7 (5) (1990) 477-492.

[8] Degiovanni M., Giannoni F., Dynamical systems with Newtonian type potentials, Ann. Sc. Norm. Sup. Pisa Cl. Sci. 15 (1988) 467-494.

[9] Degiovanni M., Giannoni F., Marino A., Dynamical systems with Newtonian type potentials, Atti Acc. Lincei Rend. Fis. Mat. 8 (81) (1987) 271-278.

[10] Gordon W., Conservative dynamical systems involving strong forces, Trans. Amer. Math. Soc. 204 (1975) 113-135.

[11] Gordon W., A minimizing property of Keplerian orbits, Amer. J. Math. 99 (5) (1977) 961-971.

[12] Meyer K.R., Hall G.R., Introduction to Hamiltonian Dynamical Systems and the $N$-Body Problem, Springer, 1991.

[13] Moser J., Stable and Random Motions in Dynamical Systems, Princeton Univ. Press, 1973.

[14] Moser J., Siegel C.M., Lectures on Celestial Mechanics, Springer, 1971.

[15] Sbano L., Collision solutions of the planar Newtonian three-body problem are not minima of the action functional, Nonlin. Diff. Eq. Appl. (1998).

[16] Serra E., Terracini S., Collisionless periodic solutions to some three-body problems, Arch. Rat. Mech. Anal. 120 (4) (1992) 305-325.

[17] Serra E., Terracini S., Noncollision solutions to some singular minimization problems with Keplerian-like potentials, Nonlin. Anal. TMA 22 (1) (1994) 45-62. 